\title{
Estimating the Trace of the Matrix Inverse by Interpolating from the Diagonal of an Approximate Inverse
}

\author{
Lingfei $\mathrm{Wu}^{\mathrm{a}, *}$, Jesse Laeuchli ${ }^{\mathrm{a}}$, Vassilis Kalantzis ${ }^{\mathrm{b}}$, Andreas Stathopoulos $^{\mathrm{a}, *}$, Efstratios Gallopoulos $^{\mathrm{c}}$ \\ ${ }^{a}$ Department of Computer Science, College of William and Mary, Williamsburg, VA 23187, United States \\ ${ }^{b}$ Department of Computer Science, University of Minnesota, Minneapolis, MN 55455, United States \\ ${ }^{c}$ Department of Computer Engineering and Informatics, University of Patras, Patras, Greece
}

\begin{abstract}
A number of applications require the computation of the trace of a matrix that is implicitly available through a function. A common example of a function is the inverse of a large, sparse matrix, which is the focus of this paper. When the evaluation of the function is expensive, the task is computationally challenging because the standard approach is based on a Monte Carlo method which converges slowly. We present a different approach that exploits the pattern correlation, if present, between the diagonal of the inverse of the matrix and the diagonal of some approximate inverse that can be computed inexpensively. We leverage various sampling and fitting techniques to fit the diagonal of the approximation to the diagonal of the inverse. Depending on the quality of the approximate inverse, our method may serve as a standalone kernel for providing a fast trace estimate with a small number of samples. Furthermore, the method can be used as a variance reduction method for Monte Carlo in some cases. This is decided dynamically by our algorithm. An extensive set of experiments with various technique combinations on several matrices from some real applications demonstrate the potential of our method.
\end{abstract}

Keywords: Matrix trace, Monte Carlo method, variance reduction, preconditioner, fitting, interpolation

\section{Introduction}

Computing the trace of a matrix $A$ that is given explicitly is a straightforward operation. However, for numerous applications we need to compute the trace of a matrix that is given implicitly by its action on a vector $x$, i.e., $A x$. Specifically, many applications are interested in computing the trace of a function of a matrix $F(A)$. Examples include estimating parameters in image restoration using the generalized cross-validation approach [1], exploring the inverse covariance matrix in uncertainty quantification [2, 3], computing observables in lattice quantum chromodynamics (LQCD) [4], or counting triangles in large graphs [5]. The matrix $A$ is large, and often sparse, so its action $F(A) x$ is typically computed through iterative methods. Because it is challenging to compute $F(A)$ explicitly, the Monte Carlo (MC) approach has become the standard method for computing the trace by averaging samples of the bilinear form $x^{T} F(A) x[6,7]$. The main purpose of this paper is to develop practical numerical techniques to address the computation of the trace of the inverse of a large, sparse matrix. But our technique can also be adapted to other functions such as the trace of the logarithm (yielding the determinant) or the trace of the matrix exponential.

For small size problems, computing $A^{-1}$ through a dense or sparse LDU decomposition is the most efficient and accurate approach $[8,9]$. This works well for discretizations of differential operators in low dimensions but becomes intractable in high dimensional discretizations. For larger size problems, domain

* Corresponding author

Email addresses: lwu@email.wm.edu (Lingfei Wu), andreasecs.wm. edu (Andreas Stathopoulos) 
decomposition and divide and conquer strategies are more tractable but still expensive [10]. In many cases, however, a low accuracy approximation is sufficient. Numerous methods have been presented to address this need for estimating the trace of the inverse of symmetric positive definite matrices through Gaussian bilinear forms [7, 11], modified moments [12, 13], and Monte Carlo (MC) techniques [7, 11, 14, 15, 12, 6, 16].

MC methods for computing the trace of a matrix are based on the structure of the Hutchinson method [1], which iteratively computes an average of matrix bilinear forms with random vectors. Variants of MC estimators are mainly analyzed and compared based on the variance of one sample $[6,17]$, which depends on the choice of the selected random vectors. For real matrices, choosing random vectors having each element \pm 1 with equal probability is known to minimize variance over all other choices of random vectors $[1,6]$ and therefore has been widely used in many applications. For complex matrices, the same result holds for vectors with $\pm 1, \pm i$ elements. In [6], Avron and Toledo analyze the quality of trace estimators through three different metrics such as trace variance, $(\varepsilon, \delta)$-approximation of the trace, and the number of random bits for different choices of random vectors. In [17], Khorasani and Ascher improve the bounds of $(\varepsilon, \delta)$-approximation for the Hutchinson, Gaussian and unit vector estimators.

There has been a number of efforts to combine the Hutchinson method with well-designed vectors based on the structure of the matrix [14, 15, 18,4]. In [15], the authors use columns of the Hadamard matrix, rather than random vectors, to systematically capture certain diagonals of the matrix. Then, the MC iteration achieves the required accuracy by continuously annihilating more diagonals with more Hadamard vectors. However, the location of the nonzeros, or of the large elements of $A^{-1}$, often does not coincide with the diagonals annihilated by the Hadamard vectors. In [18], graph coloring and probing vectors are used to identify and exploit special structures, such as bandedness or decaying properties in the elements of $A^{-1}$, to annihilate the error contribution from the largest elements. However, if the error for the chosen number of colors is large, all work has to be discarded and the probing procedure repeated until the accuracy is satisfied. In [4], the authors introduce hierarchical probing on lattices to avoid the previous problems and achieve the required accuracy in an incremental way. For all these approaches, the approximation error comes from non-zero, off-diagonal elements that have not been annihilated yet. A different MC method samples elements of the main diagonal of the $A^{-1}$ to estimate the trace. However, its variance depends on the variance of the diagonal which could be much larger than the variance of the Hutchinson method [6]. This paper focuses on this type of method with the extra assumption that an approximation to the main diagonal of $A^{-1}$ is available.

Our motivation for focusing only on the main diagonal is that the trace of $A^{-1}$ is simply a summation of a discrete, 1-D signal of either the eigenvalues or the diagonal elements of $A^{-1}$. Although we cannot compute all the diagonal elements, we may have an approximation to the whole signal from the diagonal of an approximation of $A^{-1}$ (e.g., of a preconditioner). If the two diagonals have sufficiently correlated patterns, fitting methods can be used to refine the approximation both for the diagonal and the trace. Therefore, the proposed method may serve as a standalone kernel for providing a good trace estimate with a small number of samples. But it can also be viewed as a preprocessing method for stochastic variance reduction for MC in cases where the variance reduces sufficiently. This can be monitored dynamically by our method.

We present several techniques that improve the robustness of our method and implement dynamic error monitoring capabilities. Our extensive experiments show that we typically obtain trace estimates with much better accuracy than other competing methods, and in some cases the variance is sufficiently reduced to allow for further improvements through an MC method.

\section{Preliminaries}

We denote by $\|$.$\| the 2-norm of a vector or a matrix, by N$ the order of $A$, by $Z$ an approximation of $A$, by $D$ the diagonal elements of $A^{-1}$, by $M$ the diagonal elements of $Z^{-1}$, by $\operatorname{Tr}(F(A))$ the trace of the matrix $F(A)$, and by extension, $\operatorname{Tr}(D)$ the sum of the elements of the vector $D$, by $T_{e_{i}}(F(A))$ the MC trace 
estimator of $F(A)$ using unit (orthocanonical) vectors, by $T_{Z_{2}}(F(A))$ the MC trace estimator of $F(A)$ using Rademacher vectors, by $\operatorname{diag}($.$) the diagonal operator of a matrix, and by \operatorname{Var}($.$) the variance operator of a$ random variable or a vector.

\subsection{Hutchinson trace estimator and unit vector estimator}

The standard MC method to estimate the trace of the matrix inverse is due to Hutchinson [1]. It estimates the $\operatorname{Tr}\left(A^{-1}\right)$ by averaging $s$ bilinear forms with random vectors $z_{j} \in Z_{2}^{N}=\{z(i)= \pm 1$ with probability 0.5$\}$,

$$
T_{Z_{2}}\left(A^{-1}\right)=\frac{1}{s} \sum_{j=1}^{s} z_{j}^{T} A^{-1} z_{j}
$$

The variance of this method is given by

$$
\operatorname{Var}\left(T_{Z_{2}}\left(A^{-1}\right)\right)=\frac{2}{s}\left\|A^{-1}\right\|_{F}^{2}-\frac{2}{s} \sum_{i=1}^{N}\left\|D_{i}\right\|^{2}
$$

The variance of this trace estimator is proven to be minimum over all vectors with real entries [1]. The confidence interval of a MC method reduces as $O\left(\sqrt{\operatorname{Var}\left(T_{Z_{2}}\left(A^{-1}\right)\right)}\right)$ for the given matrix.

The unit vector estimator uniformly samples $s$ vectors from the orthocanonical basis $\left\{e_{1}, \ldots, e_{N}\right\}$ [6],

$$
T_{e_{i}}\left(A^{-1}\right)=\frac{N}{s} \sum_{j=1}^{s} e_{i_{j}}^{T} A^{-1} e_{i_{j}}
$$

where $i_{j}$ are the random indices. The variance of the unit vector estimator is given by

$$
\operatorname{Var}\left(T_{e_{i}}\left(A^{-1}\right)\right)=\frac{N^{2}}{s} \operatorname{Var}(D) .
$$

The variance of the Hutchinson method depends on the magnitude of the off-diagonal elements. It converges in one step for diagonal matrices and rapidly if $A^{-1}$ is highly diagonal dominant. On the other hand, the variance of the unit vector estimator depends only on the variance of the diagonal elements. It converges in one step if the diagonal elements are all the same and rapidly if the diagonal elements are similar. Thus, the method of choice depends on the particular matrix.

\subsection{Reducing stochastic variance through matrix approximations}

Given an approximation $Z \approx A$, for which $Z^{-1}$ and $\operatorname{Tr}\left(Z^{-1}\right)$ are easily computable, we can decompose

$$
\operatorname{Tr}\left(A^{-1}\right)=\operatorname{Tr}\left(Z^{-1}\right)+\operatorname{Tr}(E),
$$

where $E=A^{-1}-Z^{-1}$. We hope that by applying the MC methods on $E$ instead on $A^{-1}$, the variance of the underlying trace estimator, in (2) or (4), can be reduced, thereby accelerating the convergence of MC. Among many ways to obtain a $Z$, we focus on the following two.

The first approach is when $Z^{-1}=(L U)^{-1}$, where the $L, U$ matrices stem from an incomplete LU (ILU) factorization of $A$, one of the most commonly used preconditioners. If the ILU is sufficiently accurate, then $M=\operatorname{diag}\left(Z^{-1}\right)$ may be a good approximation to $D$. To obtain the vector $M$ without computing the entire $Z^{-1}$, we can use an algorithm described in [19]. This algorithm requires the computation of only those entries $Z_{i j}^{-1}$ for which $L_{i j}$ or $U_{i j} \neq 0$. If the $L, U$ factors are sufficiently sparse or structured, this computation can be performed efficiently (see [20] for an example in the symmetric case).

The second approach is a low rank approximation $Z^{-1}=V \Sigma^{-1} U^{T}$, where $\Sigma$ is a diagonal matrix with the $k \ll N$ smallest singular values of $A$, and $U$ and $V$ are the corresponding left and right singular vectors. 
Eigenvalues and eigenvectors can be used instead but we do not consider it in this paper. This subspace can be obtained directly by an iterative eigensolver $[21,22]$ as a preprocessing step. The cost of this procedure is relatively small since the singular space is not needed in high accuracy. Alternatively, the space can be approximated by methods such as eigCG [23] or eigBiCG [24] while solving linear systems of equations during the MC method. This incremental approach adds only minimal overhead to the iterative linear solver but it cannot compute as many and as good quality singular vectors as the first approach. The quality of the approximation of $A^{-1}$ by $Z^{-1}$ depends on the separation of the computed singular space. Therefore, depending on the matrix, more singular triplets may be needed for a good low rank approximation. On the other hand, this space can also be used to deflate and thus accelerate subsequent linear systems. Once the singular space is computed, each diagonal element can be obtained with a single inner product of short vectors, $M_{i}=V(i,:)^{T} U(i,:) / \sigma_{i}$, and thus it is computationally inexpensive. Finally, the incremental SVD approach requires some special algorithmic attention during our algorithm, which will be pointed out later.

A computationally inexpensive, albeit less accurate approach for computing an approximation $M$ is based on variational bounds on the entries of $A^{-1}[7,25]$. Upper and lower bounds on the $i$-th diagonal entry $A_{i i}^{-1}$ are derived inexpensively since they only depend on estimates of the smallest and largest algebraic eigenvalues, $\lambda_{1}, \lambda_{N}$, and the entries of $A$. The bounds apply to both symmetric and unsymmetric matrices. For the case of a real symmetric $A$, we have [25],

$$
\frac{1}{\lambda_{N}}+\frac{\left(\lambda_{N}-A_{i i}\right)^{2}}{\lambda_{N}\left(\lambda_{N} A_{i i}-s_{i i}\right)} \leq\left(A^{-1}\right)_{i i} \leq \frac{1}{\lambda_{1}}-\frac{\left(A_{i i}-\lambda_{1}\right)^{2}}{\lambda_{1}\left(s_{i i}-\lambda_{1} A_{i i}\right)}
$$

where $s_{i j}=\sum_{k=1}^{N} A_{i k} A_{k j}$. However the bounds in (6) will not be sharp especially the upper bound [12] and the error in the approximation can be large.

In general, unless $Z^{-1}$ is highly accurate, we do not expect $\operatorname{Tr}(M)$ to be close to $\operatorname{Tr}\left(A^{-1}\right)$. However, the patterns of $M$ and $D$ often show some correlation. We demonstrate this for two example matrices, delsq50 and orsreg2205, in Figures 1 and 2 using ILU and SVD respectively. For matrix orsreg2205, both the ILU and SVD approaches return an approximate diagonal $M$ which captures the pattern of $D$ very well, with the $M$ returned by ILU being slightly better than the one from SVD. For matrix delsq50, SVD clearly captures the pattern of $D$ better than what ILU does. As in preconditioning for linear systems of equations, the appropriate approximation technique depends on the given matrix.

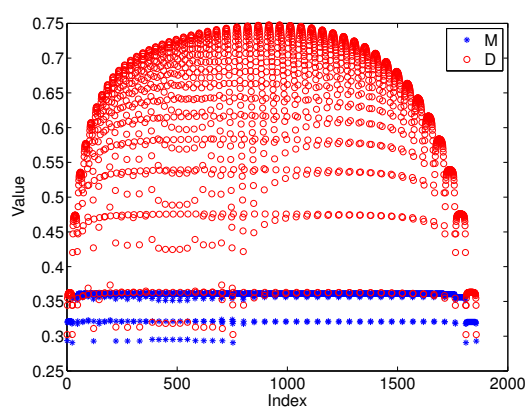

(a) $M$ does not captures pattern of $D$

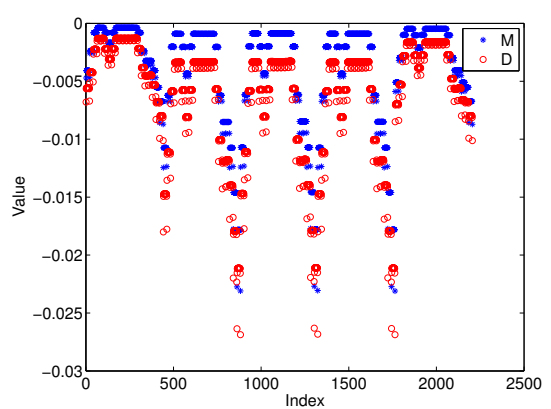

(b) $M$ quite close to $D$ and captures pattern

Figure 1: The pattern correlation between the diagonals of $A^{-1}$ and its approximation $Z^{-1}$ computed by ILU(0) on matrices (a) delsq50 and (b) orsreg2205. delsq50 is created in MATLAB by delsq (numgrid $\left.\left({ }^{\prime} S^{\prime}, 50\right)\right)$. 


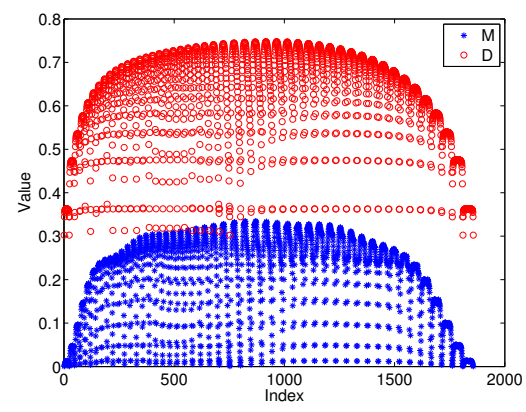

(a) $M$ not close to $D$ but captures pattern

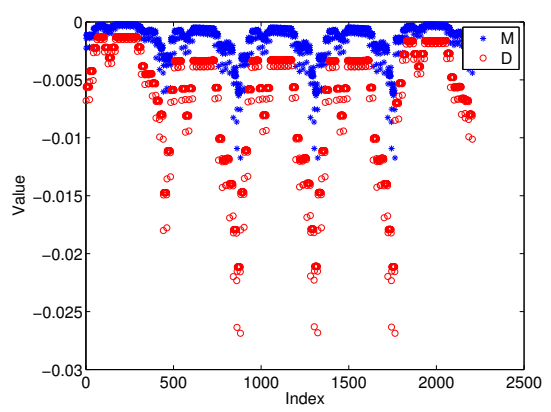

(b) $M$ not close to $D$ but captures pattern

Figure 2: The pattern correlation between the diagonals of $A^{-1}$ and its SVD approximation $Z^{-1}$ computed from the 20 smallest singular triplets of $A$ on matrices (a) delsq50 and (b) orsreg2205. delsq50 is created in MATLAB by delsq (numgrid (' $\left.\mathrm{S}^{\prime}, 50\right)$ ) .

\subsection{Comparison of different $M C$ methods and discussion on importance sampling}

Based on (2-4), we express the variance of the trace estimators $T_{Z_{2}}(E)$ and $T_{e_{i}}(E)$ as follows:

$$
\begin{gathered}
\operatorname{Var}\left(T_{Z_{2}}(E)\right)=\frac{2}{S}\|E\|_{F}^{2}-\frac{2}{s} \sum_{i=1}^{N}\|\operatorname{diag}(E)\|^{2}, \\
\operatorname{Var}\left(T_{e_{i}}(E)\right)=\frac{N^{2}}{s} \operatorname{Var}(\operatorname{diag}(E)) .
\end{gathered}
$$

Figures 1 and 2 show there is potential for the variances of $T_{Z_{2}}(E)$ or $T_{e_{i}}(E)$ to be smaller than those of $T_{Z_{2}}\left(A^{-1}\right)$ and $T_{e_{i}}\left(A^{-1}\right)$. However, for a given matrix, we must gauge which MC method would be better, and whether the variances need further improvement.

The estimator $T_{e_{i}}(E)$ has the interesting property that if $M=D+c$, where $c$ is a constant, then its variance in (8) is zero and we obtain the correct trace in one step. Although we cannot expect this in practice, it means that the shift observed between $M$ and $D$ in Figure 2(a) should not affect the effectiveness of $T_{e_{i}}(E)$.

On the other hand, $T_{e_{i}}(E)$ fails to identify correlations of the form $M=c D$. For such cases, importance sampling is preferred, where $M$ plays the role of a new distribution simulating the distribution of $D$. Assume that both $D$ and $M$ have been shifted by the same shift so that $M_{i}>0$ if $D_{i}>0, i=1, \ldots, N$. To transform $M$ into a probability mass function, let $G=\frac{1}{\operatorname{Tr}(M)} M$. To obtain an estimator of the trace of $D$ with importance sampling, we replace the uniform sampling of $D_{i}$ values with sampling with probability $G_{i}[26]$. Then, instead of (3), the importance sampling estimator is:

$$
T_{I S}(D)=\frac{N}{s} \sum_{j=1}^{s} D_{i_{j}} \frac{\frac{1}{N}}{G_{i_{j}}}=\frac{\operatorname{Tr}(M)}{s} \sum_{j=1}^{s} \frac{D_{i_{j}}}{M_{i_{j}}} .
$$

When $M=c D$, the variance of $T_{I S}(D)$ is zero and it finds the trace in one step. However, it completely fails to identify shift correlations. In general $D$ and $M$ may have a more complex relationship that neither $T_{I S}(D)$ or $T_{e_{i}}(E)$ can capture. This motivates our idea to explore general fitting models to approximate $D$.

\section{Approximating the trace of a matrix inverse}

We seek to construct a function $f$, such that $D \approx f(M)$. Then we can decompose

$$
\operatorname{Tr}\left(A^{-1}\right)=\operatorname{Tr}(D-f(M))+\operatorname{Tr}(f(M)) .
$$


$\operatorname{Tr}(f(M))$ is trivially computed for a given $f$. A key difference between the approaches in (10) and (5) is that it is easier to find a fitting of the two vectors $M$ and $D$ if a strong pattern correlation exists between them than to fit all corresponding elements in matrices $A^{-1}$ and $Z^{-1}$. If $\operatorname{Tr}(f(M))$ is a good approximation to $\operatorname{Tr}\left(A^{-1}\right)$ and its accuracy can be evaluated easily, we can directly use this quantity; Otherwise, we can apply the unit vector MC estimator to compute $\operatorname{Tr}\left(E_{f i t}\right)=\operatorname{Tr}(D-f(M))$, provided that its variance

$$
\operatorname{Var}\left(T_{e_{i}}\left(E_{f i t}\right)\right)=\frac{N^{2}}{s} \operatorname{Var}\left(E_{f i t}\right)
$$

is smaller than the variances in (2), (3), (7) and (8).

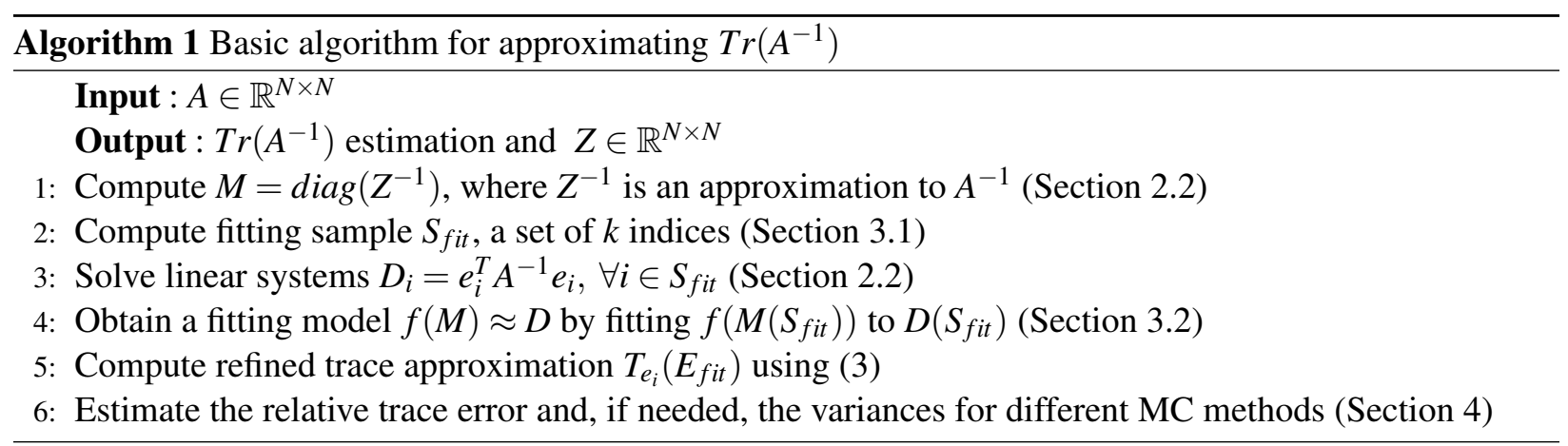

The basic description of the proposed estimator is outlined in Algorithm 1. First, our method computes an approximation $M$ of $D$ using one of the methods discussed in the previous section. If that method is based on a preconditioner or a low rank approximation, that preconditioner $Z^{-1}$ could also be used to speed up the solution of linear systems in step 3. Second, it finds a fitting sample $S_{f i t}$, a set of indices that should capture the important distribution characteristics of $D$. Since we have no information about $D$, Section 3.1 discusses how to tackle this task by considering the distribution of $M$. Third, it computes the values of $D\left(S_{f i t}\right)$ by solving the corresponding linear systems using a preconditioned iterative solver. Since this is the computational bottleneck, the goal is to obtain good accuracy with far fewer fitting points than the number of vectors needed in MC. Fourth, it computes a fitting model that has sufficient predictive power to improve the diagonal approximation. This critical task is discussed in Section 3.2. Finally, since there are no a-posteriori bounds on the relative error of the trace, we use a combination of statistical approaches and heuristics to estimate it incrementally at every step. If the estimated error is not sufficiently small, our method can be followed by an MC method. For this reason, the algorithm also estimates dynamically the variances of the two different MC estimators (7) and (8) so that the one with the smallest variance is picked. The dynamic evaluation of error and variances is discussed in Section 4.

\subsection{Point Identification Algorithm}

We need to identify a set of indices $S_{f i t}$ based on which we can compute a function $f$ that fits $f\left(M\left(S_{f i t}\right)\right)$ to $D\left(S_{f i t}\right)$ so that $|\operatorname{Tr}(f(M))-\operatorname{Tr}(D)|$ is minimized. It is helpful to view $\operatorname{Tr}(D)$ as an integral of some hypothetical one dimensional function which takes the values $D(i)=D_{i}$ on the discrete points $i=1, \ldots, n$. Our goal is to approximate $\int_{0}^{N} D(x) d x$ with far fewer points than $n$. Because it is one dimensional, it may be surprising that the Monte Carlo approach can be advantageous than numerical integration. However, the effectiveness of any numerical quadrature rule relies on the smoothness of the function to be integrated, specifically on the magnitude of its higher order derivatives. In our case, our hypothetical function may have no smoothness or bounded derivatives. More practically, we deal with a set of discrete data points in $D$ for which smoothness must be defined carefully. A typical definition of smoothness for discrete data is based on the Lipschitz continuity, i.e., $\left|D_{i}-D_{j}\right|<c|i-j|$ [27]. The lower the constant $c$, the smoother the 
set of data. Hence, for a continuous function, the larger the magnitude of its first derivative, the less smooth its discretized points are. In the context of integrating an arbitrary $D$, the first order divided difference (i.e., the discretized first derivative) $D_{i}-D_{i+1}$ may be arbitrarily large in magnitude and therefore numerical integration may not work any better than random averaging.

Even for matrices that model physically smooth phenomena (e.g., in PDEs), the matrix may be given in an ordering that does not preserve physical locality.

Consider a sorted permutation of the diagonal, $\hat{D}=\operatorname{sort}(D)$. Obviously $\operatorname{Tr}(D)=\operatorname{Tr}(\hat{D})$, but $\hat{D}$ is monotonic and, based on the definition of Lipschitz discrete smoothness, it is maximally smooth, i.e.,

$$
|\hat{D}(i)-\hat{D}(j)| \leq \Delta|i-j|
$$

for the smallest possible $\Delta \in \mathbb{R}_{+}$among all permutations of $D^{1}$. Monotonicity implies that, in the absence of any additional information about the data, a simple trapezoidal rule minimizes the worst case integration error [28]. In addition, if we are allowed to choose the integration points sequentially, based on the points computed so far, then a much better average case error can be obtained [29, 30]. On the other hand, if bounds are known on the discrete smoothness of $\hat{D}$, better worst case error bounds can be established. Since $D$, however, is not available, we turn to its approximation $M$.

A close pattern correlation between $M$ and $D$ means that the elements of $M$ should have a similar distribution as those of $D$, or that $\hat{M}=\operatorname{sort}(M)$ should be similar to $\hat{D}$. Let us assume for the moment, that the index that sorts $D$ to $\hat{D}$ sorts also $M$ to $\hat{M}$. In other words, we assume a complete correlation between the monotonic orderings of $M$ and $D$ even though their values may differ. Then, we can work on the surrogate model $\hat{M}$ for which we can afford to identify the best quadrature points that yield the smallest error in $\operatorname{Tr}(\hat{M})$. These will be the ideal points over all permutations of $M$ for computing the fitting function $f$.

Specifically, we need to select indices that capture the important distribution changes in $\hat{M}$. For example, identifying minimum and maximum elements of $M$ sets the range of approximation for $f$ and avoids extrapolation. We also look for entries in $\hat{M}$ that deviate highly from their neighbors (where the first order divided difference of $\hat{M}$ and hopefully of $\hat{D}$ has a large value). This strategy has three advantages. First, between such indices the data is smooth so the integral $\operatorname{Tr}(\hat{M})$ should be captured well by the trapezoidal rule. Second, and more important, we can obtain a more accurate fitting function $f$ in a piecewise manner in intervals where $\hat{D}$ has similar behavior. Third, in this way we avoid picking points with the same value $\hat{M}_{i}=\hat{M}_{j}$ which can create problems in the fitting function.

The proposed index selection method is shown in Algorithm 2. Initially the set of sampled indices, $\hat{S}_{f i t}$, includes the indices of the extrema of $\hat{M}, 1$ and $N$. Then, for every interval $(i, j)$, with $i, j$ consecutive indices in $\hat{S}_{f i t}$, we find the index $t$ from $i+1$ to $j-1$ that minimizes the trapezoidal rule error for computing $\operatorname{Tr}(\hat{M}(i: j))$ :

$$
\underset{i<t<j, t \in \mathbb{Z}}{\operatorname{argmin}}(\mid \hat{M}(i)-\hat{M}(j)) *(i-j)-(\hat{M}(i)-\hat{M}(t)) *(i-t)-(\hat{M}(t)-\hat{M}(j)) *(t-j) \mid) .
$$

The process continues until it reaches a maximum number of sampling points or until the maximum error over all intervals decreases by a fixed value, say 0.001 . The value of this threshold depends on how close the patterns of $M$ and $D$ are correlated, a question we had postponed and we discuss next.

The points selected by the previous algorithm minimize the error for computing $\operatorname{Tr}(M)$ with only $\left|\hat{S}_{f i t}\right|$ points. How good are these points for fitting $\hat{M}$ to $\hat{D}$ and thus obtaining a small error in $\operatorname{Tr}(D)$ ? Consider the $M$ and $D$ as discrete functions from $\{1, \ldots, n\} \rightarrow \mathbb{R}$ and assume they are bijective in their ranges. Let

\footnotetext{
${ }^{1}$ It is easy to see that $\Delta=\max _{i=2, N}|\hat{D}(i)-\hat{D}(i-1)|=\hat{D}\left(i_{0}\right)-\hat{D}\left(i_{0}-1\right)$ is the smallest value that satisfies (12) for the sorted array. Assume there is a different permutation $D^{\prime}$ that satisfies (12) with $\delta<\Delta$. If $D^{\prime}(k)=\hat{D}\left(i_{0}\right)$, then $\delta \geq\left|D^{\prime}(k)-D^{\prime}(k-1)\right| \geq$ $\hat{D}\left(i_{0}\right)-\hat{D}\left(i_{0}-1\right)=\Delta$, which contradicts the assumption.
} 
$J, G:\{1, \ldots, n\} \rightarrow\{1, \ldots, n\}$ be the two permutation functions that sort $M$ to $\hat{M}$ and $D$ to $\hat{D}$, i.e., $\hat{M}(i)=$ $M(J(i))$ and $\hat{D}(i)=D(G(i))$. Our method relies on the assumption that the distribution of $\hat{M}$ captures the distribution of $\hat{D}$, or that there exists a smooth function $f$ that can be approximated well with a low degree polynomial that fits $\hat{D}(i)=f(\hat{M}(i))$. For any $i$, there are two indices $m=G(i), k=J(i)$ with $m \neq k$ in general that satisfy,

$$
D(m)=D(G(i))=\hat{D}(i)=f(\hat{M}(i))=f(M(J(i)))=f(M(k)) .
$$

Our algorithm first picks an index $i$ for $\hat{M}$ and derives the original index $k=J(i)$ in $M$. Since the permutation $G$ is unknown, we do not know the $D(m)$ that should be matched with $M(k)$. Thus, the algorithm makes the simplifying assumption that $G=J$ and computes $D(k)$ instead. However, this corresponds to a different $\hat{D}(j)$, where $G(j)=k$. Therefore, using (12), we can bound the error for this mismatch by

$$
|\hat{D}(i)-\hat{D}(j)| \leq \Delta|i-j|=\Delta\left|J^{-1}(k)-G^{-1}(k)\right| .
$$

This implies that as long as the permutations $J$ and $G$ are locally similar, or in other words they do not shuffle the same index of $M$ and $D$ too far from each other, the error is small and therefore the fitting should work well. On the other hand, if $M$ is a random permutation of $D$, there is no good fitting, even though $\hat{M}=\hat{D}$. This implies that the traces of $f(M)$ and $D$ may be very close but not their individual elements. It also means that its variance of $E_{f i t}$ may not always be small (see the examples in the following section as well as in Section 4.2).

In practice, since we do not know the permutation $G$, it is possible that some flat area of $\hat{M}$ is associated with important changes in $\hat{D}$. To alleviate the effect of a possible local pattern mismatch, we instead empirically insert the midpoint of the current largest interval every $k$ samples (we choose $k=5$ in lines 11-14). Returning to the choice of threshold in the algorithm, we see that going below a threshold helps the accuracy of $\operatorname{Tr}(M)$ but not necessarily of $\operatorname{Tr}(D)$. The reason is that the greedy point selection strategy becomes less effective in determining the local mismatching patterns. Therefore, we terminate searching for a new index based on (13) if the maximum error is less than 0.001 . We found this threshold to be sufficient in our experiments. If the maximum required samples have not been generated, we continue by simply bisecting the largest intervals until maxPts is reached (in lines 16-18). On exit, we compute $S_{f i t}$ which maps $\hat{S}_{f i t}$ to the original unsorted ordering.

A typical sampling result produced by our method is shown in Figure 3. The graph on the left shows the diagonals $M$ and $D$ plotted in their original order. The pattern correlation between them is clear, but it is less straightforward how to pick the fitting points. Figure 3(b) shows both diagonals plotted with the order of indices that sorts $\hat{M}$, i.e., $M(J), D(J)$. The points picked by our algorithm based on $\hat{M}$ correspond to an almost monotonic sequence of points in $D(J)$. The associated indices can then be used to identify a suitable set of entries in $D$ to perform the numerical integration.

\subsection{Two Fitting Models}

Next we construct a fitting model that minimizes $\left\|f\left(M\left(S_{f i t}\right)\right)-D\left(S_{f i t}\right)\right\|$. The fitting model must have sufficient predictive power with only a small number of points and it should avoid oscillating behavior since we work on the monotonic sequence $\hat{M}$ which we assume correlates well with $\hat{D}$. Therefore, we consider a linear model and a piecewise polynomial model.

The MC methods in (5) and (10) can resolve the trace when $D=M+c$ while importance sampling can resolve the trace when $D=c M$. To combine these, we first use a linear model, $y=b M+c$. We determine the parameters $b, c$ by a least squares fitting, $\operatorname{argmin}_{b, c \in \mathbb{R}}\left\|D\left(S_{f i t}\right)-\left(b M\left(S_{f i t}\right)+c\right)\right\|_{2}$. The linear model may be simple but avoids the large oscillations of higher degree polynomials, and in many cases it is quite effective in improving the accuracy of the trace estimation and reducing the variance of the diagonal elements of $E_{f i t}$. The linear fitting algorithm is described in Algorithm 3. Figure 4(a) shows the fitting result on the example matrix of the previous section, in the original order of $D$. 


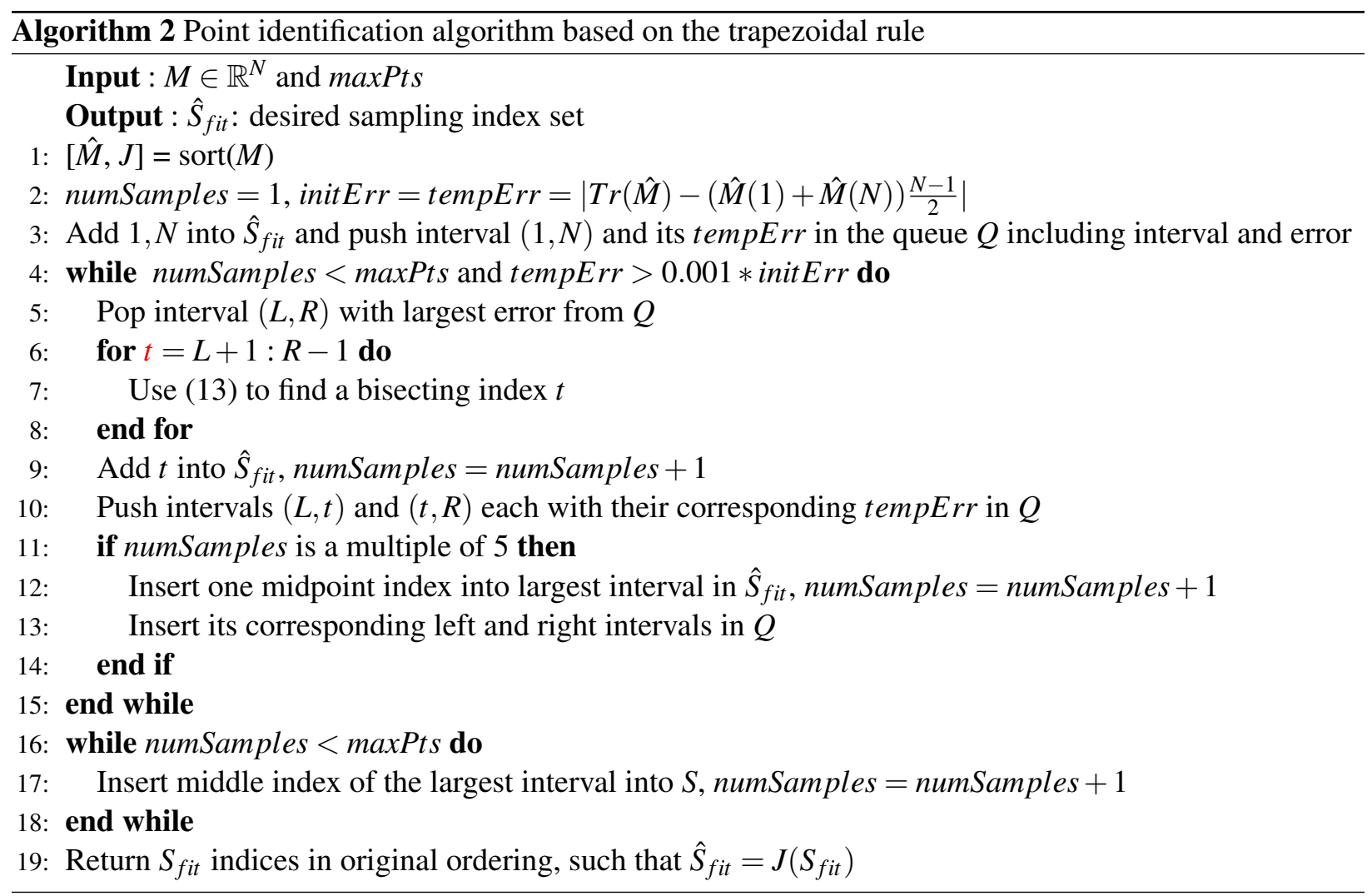

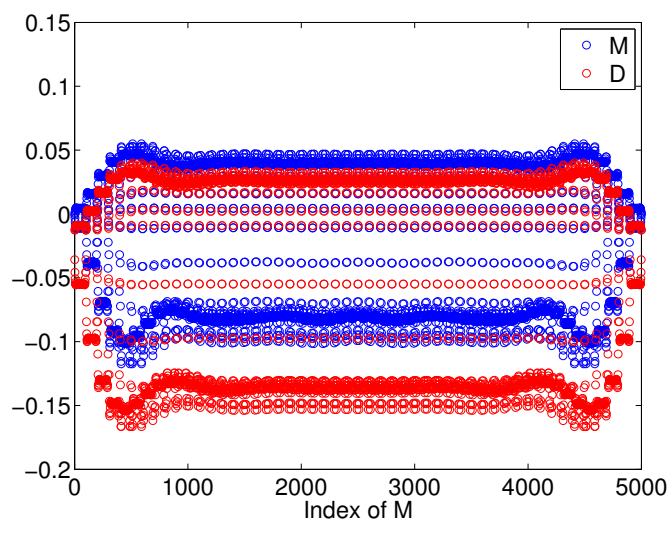

(a) Distribution of elements of $M$ and $D$

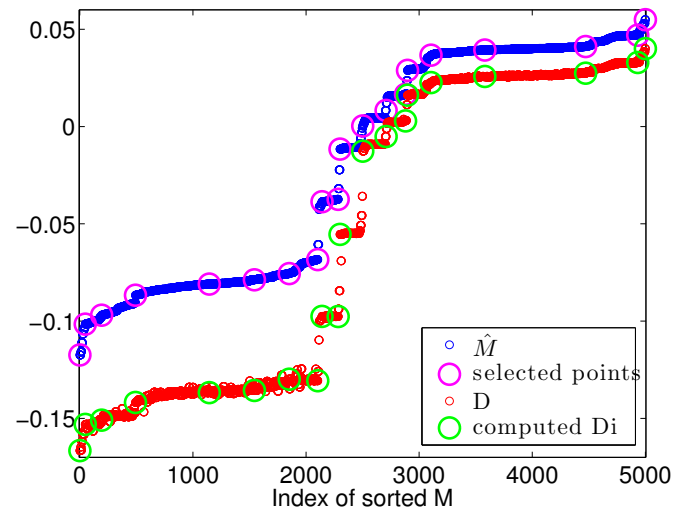

(b) Sample points for fitting

Figure 3: A typical example to show our sampling strategy based on the pattern correlation of $M$ and $D$. $M$ is computed by 20 singular vectors of matrix RDB5000. In the right figure, the magenta and green circles denote the sample points associated with the sampling indices $S_{f i t}$ in $\hat{M}$ and $D$.

The linear model preserves the shape of $M$, and therefore relies exclusively on the quality of $M$. To take advantage of our premise that the distribution $\hat{M}$ approximates well the distribution of $\hat{D}$, our next fitting model is the Piecewise Cubic Hermite Spline Interpolation (PCHIP). It was proposed in [31] to construct a visually pleasing monotone piecewise cubic interpolant to monotone data. The PCHIP interpolant is only affected locally by changes in the data and, most importantly, it preserves the shape of the data and respects monotonicity. Therefore, we work on $\hat{M}$ and the indices $\hat{S}_{f i t}=\left[1=s_{1}, s_{2}, \ldots, s_{k-1}, s_{k}=N\right]$ which are given 


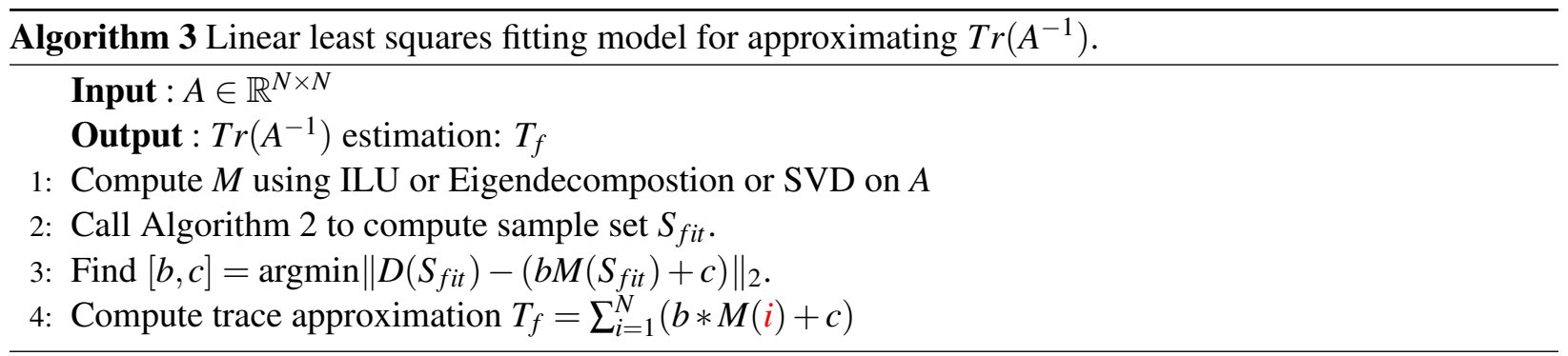

in an order such that $\alpha=\hat{M}\left(s_{1}\right) \leq \hat{M}\left(s_{2}\right) \leq \cdots \leq \hat{M}\left(s_{k}\right)=\beta$ is a partition of the interval $[\alpha, \beta]$. An index $s_{i}$ corresponds to the index $J^{-1}\left(s_{i}\right)$ in the original ordering of $M$, where $J$ is from Algorithm 2 and $J^{-1}$ denotes the inverse mapping of the sorted list. Thus, for each $s_{i}$ we compute $D\left(J^{-1}\left(s_{i}\right)\right), i=1, \ldots, k$, and we use PCHIP to construct a piecewise cubic function such that,

$$
p\left(\hat{M}\left(s_{i}\right)\right)=D\left(J^{-1}\left(s_{i}\right)\right), \quad i=1,2, \ldots, k .
$$

Notice that $p(x)$ will be monotone in the subintervals where the fitting points $D\left(J^{-1}\left(s_{i}\right)\right)$ are also monotone. Therefore, as long as $M$ is close to $D$ in the sense of (14), integration of $p(x)$ will be very accurate.

The PCHIP model is given in Algorithm 4. The first two steps are the same as in Algorithm 3. In step 3, we apply the function unique to remove the duplicate elements of $\left.\hat{M}\left(\hat{S}_{f i t}\right)\right)$ to produce a sequence of unique values as required by PCHIP. This yields a subset of the indices, $\hat{S}_{f i t}^{\prime}$, which is mapped to original indices as $I=J^{-1}\left(\hat{S}_{f i t}^{\prime}\right)$ to be used in PCHIP.

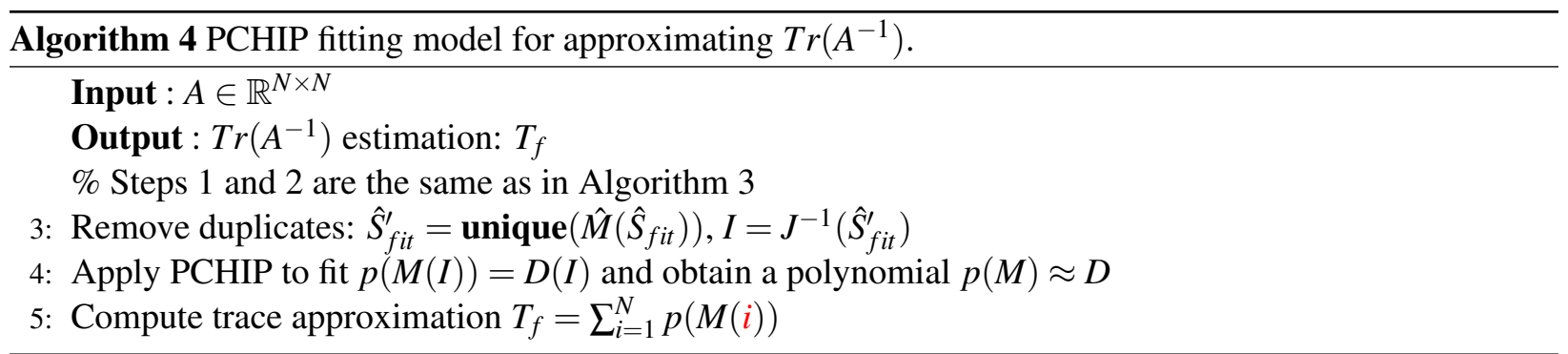

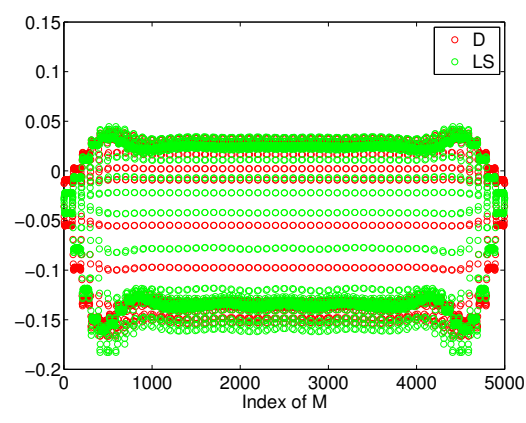

(a) Linear LS model in original order

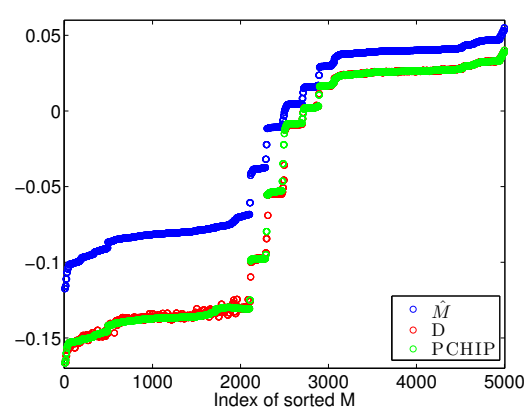

(b) PCHIP model in sorted order

Figure 4: Fitting results of the matrix RDB5000 in original order and sorted order with linear LS model and PCHIP model.

Figure 4(b) shows the result of fitting on the RDB5000 example of the previous figures. Table 1 compares the relative error for the trace, as well as the variances of $D, D-M$ and $D-p(M)$ when using the linear LS and PCHIP models on two test matrices, OLM5000 and KUU. In both cases, the two models can provide 
a trace estimate of surprising relative accuracy of the order of $1 e-2$ with only 20 fitting points. In addition, for matrix OLM5000, the standard deviation of MC on $D-p(M)$ is reduced by a factor of 10 compared to that of MC on $D$ (a speedup of 100 in terms of samples). However, for matrix KUU, the standard deviation of MC on $D-p(M)$ does not improve much over MC on $D$. This reflects the discussion in Section 3.1, and suggests that our method can serve as a standalone kernel for giving a fast trace estimate. In addition, in some cases the method can reduce the variance significantly to accelerate a second stage MC.

Table 1: Comparing the trace relative error and variances in (4), (8) and (11) for matrices OLM5000 and KUU, using the linear LS and PCHIP models with 20 fitting points each. The traces of matrix OLM5000 and KUU are $\operatorname{Tr}\left(A^{-1}\right)=-5.0848 e+02$ and $3.6187 e+03$, respectively.

\begin{tabular}{|c|c|c|c|c|c|c|}
\hline \multicolumn{2}{|l|}{ Matrix } & \multicolumn{2}{|l|}{ OLM5000 } & \multicolumn{3}{|c|}{ KUU } \\
\hline Model & M & LS & PCHIP & M & LS & PCHIP \\
\hline Relative error & $9.6650 \mathrm{e}-01$ & $2.2320 \mathrm{e}-02$ & $1.4288 \mathrm{e}-02$ & $5.8316 \mathrm{e}-01$ & $1.2207 \mathrm{e}-02$ & $1.4469 \mathrm{e}-02$ \\
\hline $\operatorname{Std}\left(T_{e_{i}}\left(A^{-1}\right)\right)$ & $1.1425 \mathrm{e}+02$ & $1.1425 \mathrm{e}+02$ & $1.1425 \mathrm{e}+02$ & $2.8731 \mathrm{e}+02$ & $2.8731 \mathrm{e}+02$ & $2.8731 \mathrm{e}+02$ \\
\hline $\operatorname{Std}\left(T_{e_{i}}(D-M)\right)$ & $1.1007 \mathrm{e}+02$ & $1.1007 \mathrm{e}+02$ & $1.1007 \mathrm{e}+02$ & $1.4999 e+02$ & $1.4999 \mathrm{e}+02$ & $1.4999 e+02$ \\
\hline $\operatorname{Std}\left(T_{e_{i}}(D-p(M))\right)$ & - & $2.0332 \mathrm{e}+01$ & $1.7252 \mathrm{e}+01$ & - & $1.6137 \mathrm{e}+02$ & $1.6289 \mathrm{e}+02$ \\
\hline
\end{tabular}

\section{Dynamic Evaluation of Variance and Relative Trace Error}

Since there are no a-posteriori bounds for the accuracy of our results, we develop methods that use the information from the solution of the linear systems to incrementally estimate the trace error and the variances of the resulting approximations. This approach is also useful when $M$ is updated with more left and right eigenvectors or singular vectors obtained from the solution of additional linear systems.

\subsection{Dynamic Variance Evaluation}

To decide which MC method we should use after the fitting stage or even whether it is beneficial to use the fitting process for variance reduction, we monitor incrementally the variances $\operatorname{Var}\left(T_{e_{i}}\left(A^{-1}\right)\right)$, $\operatorname{Var}\left(T_{e_{i}}\left(E_{f i t}\right)\right), \operatorname{Var}\left(T_{Z_{2}}\left(A^{-1}\right)\right)$, and $\operatorname{Var}\left(T_{Z_{2}}(E)\right)$, with the aid of the cross-validation technique [32].

Our training set is the fitting sample set $D\left(S_{f i t}\right)$, while our test set $D\left(S_{m c}\right)$ is a small random set which is independent of the fitting sample set. If we want to combine our method with MC, eventually more samples need to be computed, and thus we can pre-compute a certain number of them as the test set $D\left(S_{m c}\right)$. We have used the holdout method [33], a single train-and-test experiment for some data splitting strategy since the fitting sample set is fixed.

To compute $D\left(S_{f i t}\right)$ or $D\left(S_{m c}\right)$, a linear system with multiple right hand sides is solved as follows:

$$
A_{i i}^{-1}=e_{i}^{T} x_{i}, \quad A x_{i}=e_{i}, \forall i \in S_{f i t} \cup S_{m c} .
$$

The computed column vectors $x_{i}$ can be used to estimate the Frobenius norm of both $A^{-1}$ and $E=A^{-1}-Z^{-1}$ [34, 35]. Then $\operatorname{Var}\left(T_{Z_{2}}\left(A^{-1}\right)\right)$ and $\operatorname{Var}\left(T_{Z_{2}}(E)\right)$ can be estimated as follows:

$$
\begin{gathered}
\operatorname{Var}\left(T_{Z_{2}}\left(A^{-1}\right)\right) \approx \frac{2 N}{s^{2}} \sum_{i}\left(\left\|x_{i}\right\|^{2}-\left|D_{i}\right|^{2}\right), \forall i \in S_{f i t} \cup S_{m c}, \\
\operatorname{Var}\left(T_{Z_{2}}(E)\right) \approx \frac{2 N}{s^{2}} \sum_{i}\left(\|E(:, i)\|^{2}-|E(i, i)|^{2}\right), \forall i \in S_{f i t} \cup S_{m c},
\end{gathered}
$$


where $E(:, i)=E e_{i}$. Simultaneously, based on the sampled diagonal elements $A_{i i}^{-1}$, we can also update the evaluation of $\operatorname{Var}\left(T_{e_{i}}\left(E_{f i t}\right)\right), \operatorname{Var}\left(T_{e_{i}}(E)\right)$ and $\operatorname{Var}\left(T_{e_{i}}\left(A^{-1}\right)\right)$. Here we only show the computation of the unbiased variance estimation for $\operatorname{Var}\left(T_{e_{i}}\left(E_{f i t}\right)\right)$ by:

$$
\operatorname{Var}\left(T_{e_{i}}\left(E_{f i t}\right)\right) \approx \frac{N^{2}}{s-1} \operatorname{Var}\left(E_{f i t}\left(S_{m c}\right)\right)
$$

Note that $S_{f i t}$ should not be used for estimating the variance of the unit vector MC estimator since these sample points are exact roots of the PCHIP function.

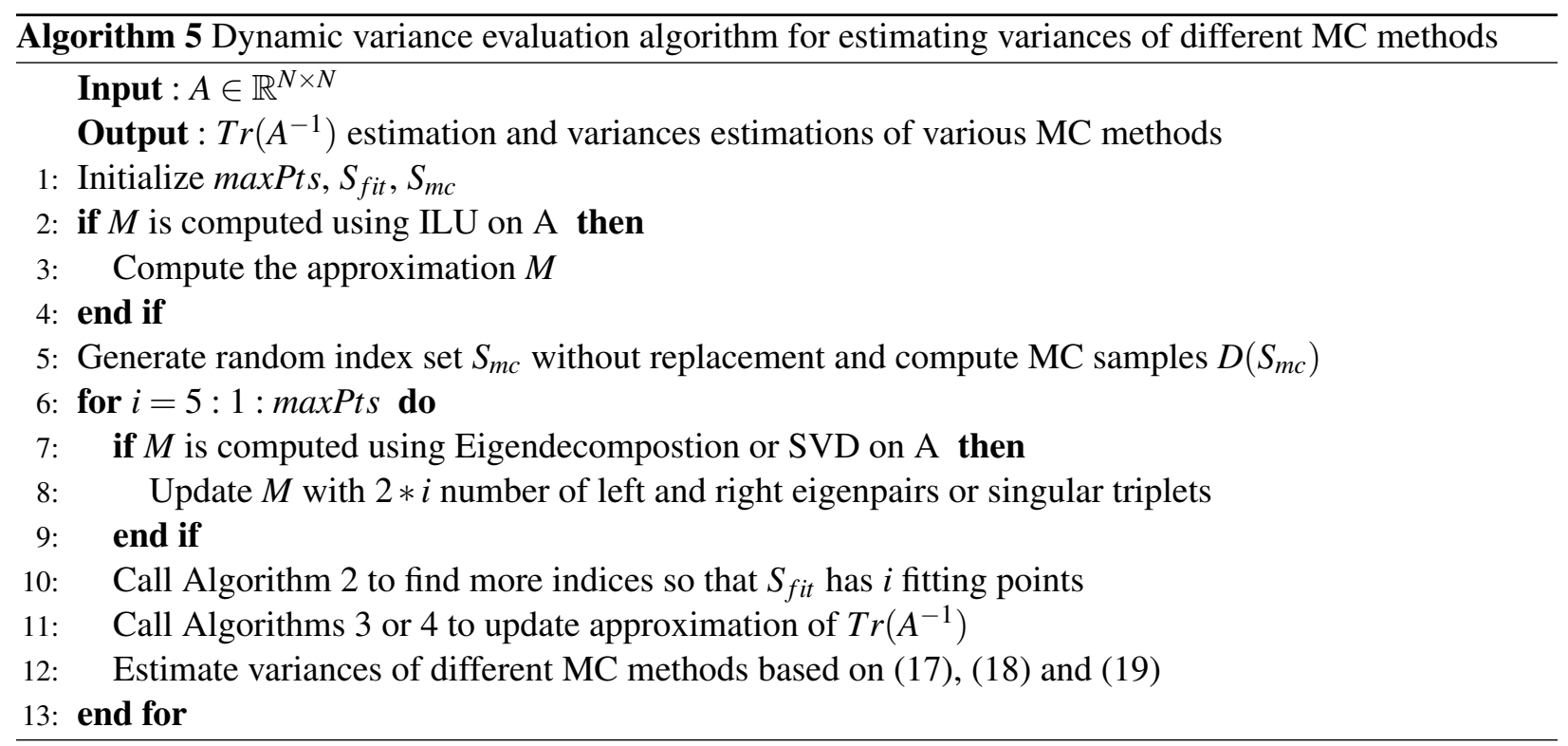

We implement the dynamic variance evaluation scheme in Algorithm 5. In lines 2-4 and 7-9, the approximation $M$ can be computed using an ILU factorization at the beginning of the procedure or be updated with increasing number of singular triplets or eigenpairs. Note that if $M$ is obtained by a partial eigendecomposition or SVD, the updated $M$ is different in two consecutive steps, and thus Algorithm 2 will return a slightly different index set $S_{f i t}$ which may not be incremental. This may not provide a consistent improvement of the relative trace error during the fitting progress. In line 10 of the algorithm, we force the points to be incremental between steps $i$ and $i+1$ as follows; we generate the entire set $S_{f i t}^{(i+1)}$ and remove the indices that lie the closest to the previous index set $S_{f i t}^{(i)}$. The remaining index set is incorporated into $S_{f i t}^{(i)}$. This simple scheme works quite well experimentally.

Figure 5 shows how the actual and the estimated variances for three MC methods match for the test matrix RDB5000. In addition, the relative difference between different MC methods becomes clear after only a few points which facilitates not only the proper choice of MC method but also an early decision to stop if further fitting is not beneficial. In the numerical experiments section we show that these results are typical for matrices from a wide variety of applications.

\subsection{Monitoring Relative Trace Error}

As discussed in Section 3.1 and showed in Table 1, the variance of MC on the vector $E_{\text {fit }}$ may not be reduced if the sorting permutations of $\hat{M}$ and $\hat{D}$ are dissimilar. Even in such cases, however, our fitting method can provide a good trace estimation with only a small number of samples. We further investigate this by comparing the elements of $D$ and $p(M)$ with different orderings. Figure 6(a) shows the elements of $p(M(J))$ and $D(J)$, i.e., with respect to the order of $\hat{M}$. It illustrates that although $p(M)$ captures the 


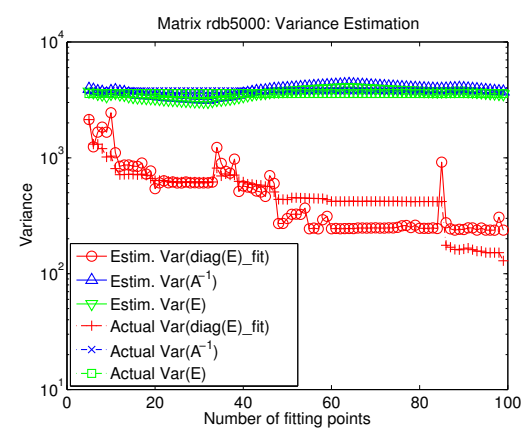

(a) Dynamic variance estimation with ILU

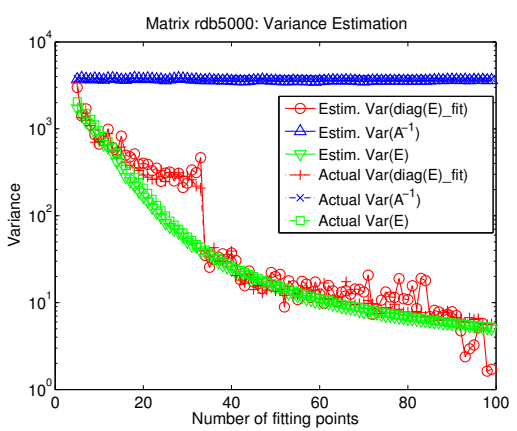

(b) Dynamic variance estimation with SVD

Figure 5: Comparing estimated variances and actual variances of unit vector on $E_{f i t}$ (denoted as $\left.\operatorname{Var}\left(\operatorname{diag}(E)_{-} f i t\right)\right)$ and Rademacher vector on $A^{-1}$ (denoted as $\operatorname{Var}\left(A^{-1}\right)$ ) and $E$ (denoted as $\operatorname{Var}(E)$ ) of the matrix RDB5000 with ILU and SVD respectively.

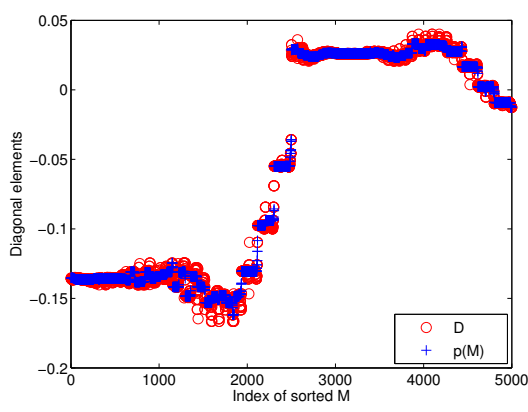

(a) Comparing $D$ and $p(M)$ in order of $\hat{M}$

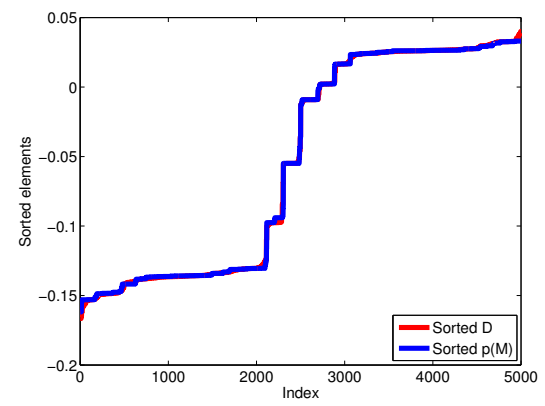

(b) Comparing sorted $D$ and sorted $p(M)$

Figure 6: Compare $D$ and $p(M)$ of the matrix RDB5000 in different order where $M$ is computed by ILU.

pattern of $D$, the order of its elements does not correspond exactly to that of $D$; hence the small reduction in $\operatorname{Var}\left(T_{e_{i}}\left(E_{f i t}\right)\right)$. However, Figure 6(b) reveals that the distributions of the sorted $p(M)$ and the sorted $D$ almost coincide; hence, the two integrals $\operatorname{Tr}(p(M))$ and $\operatorname{Tr}(D)$ are very close.

The main obstacle for using our method as a standalone kernel for trace estimation is that there is no known way to measure or bound the relative trace error. Resorting to the confidence interval computed by the variance of a MC estimator is pessimistic as our results show (see also [6, 17]). We note the similarity to the much smaller error obtained in the average case of integrating monotonic functions with adaptive bilinear forms versus the worst case known bounds [28, 29].

Motivated by these previous research results, we show how to develop practical criteria to monitor the relative trace error in our Algorithms. Suppose at each step of the fitting process in Algorithm 5 we collect a sequence of trace estimates $T_{i}, i \in[1, \max P t s]$. Consider the trace estimations in two successive steps,

$$
\frac{\left|T_{i}-T_{i+1}\right|}{\left|T_{i+1}\right|}=\frac{\left|\left(T_{i}-\operatorname{Tr}(D)\right)-\left(T_{i+1}-\operatorname{Tr}(D)\right)\right|}{\left|T_{i+1}\right|}=\frac{\left|E_{i}-E_{i+1}\right|}{\left|T_{i+1}\right|} \leq \frac{2 \max \left(\left|E_{i}\right|,\left|E_{i+1}\right|\right)}{\left|T_{i+1}\right|} \approx \frac{2 \max \left(\left|E_{i}\right|,\left|E_{i+1}\right|\right)}{|\operatorname{Tr}(D)|} .
$$

As long as $T_{i}$ converges with more fitting points, the relative difference of two successive trace estimations can serve as an approximation to the relative error. However, when the global pattern provided by $M$ and $p(M)$ is not fully matched to that of $D$, convergence of $T_{i}$ stagnates until enough points have been added to resolve the various local patterns. To determine whether the current relative trace error estimation can be trusted, we present our second heuristic by considering the error bound of our fitting models.

When approximating $f(\hat{M})$ with $p(\hat{M})$, the PCHIP Hermite cubic splines with $k$ points on the interval 
$[\alpha, \beta]$, the bound on the error $E(\hat{M})=f(\hat{M})-p(\hat{M})$ is given by [36],

$$
|E(\hat{M})| \leq \frac{1}{384} h^{4}\left\|f^{(4)}\right\|_{\infty,[\alpha, \beta]}
$$

where $h=\frac{\beta-\alpha}{k}$, and $\left\|f^{(4)}\right\|_{\infty,[\alpha, \beta]}$ denotes the maximum value of the fourth derivative of $f$ in the entire interval $[\alpha, \beta]$. Since $\left\|f^{(4)}\right\|_{\infty,[\alpha, \beta]} / 384$ is a constant, in two successive fitting steps we have,

$$
\frac{\left|E_{i}(\hat{M})\right|}{\left|E_{i+1}(\hat{M})\right|} \approx \frac{h_{i}^{4}}{h_{i+1}^{4}}=\frac{\left((\beta-\alpha) / k_{i}\right)^{4}}{\left((\beta-\alpha) / k_{i+1}\right)^{4}}=\left(\frac{k_{i+1}}{k_{i}}\right)^{4},
$$

where $k_{i}$ and $k_{i+1}$ are the number of fitting points in two consecutive steps. We can use (22) to estimate the maximum possible improvement between two consecutive trace errors. If the $i+1$ trace error estimate reduces over the $i$-th estimate by a factor of more than $\left(k_{i} / k_{i+1}\right)^{4}$, we do not trust it.

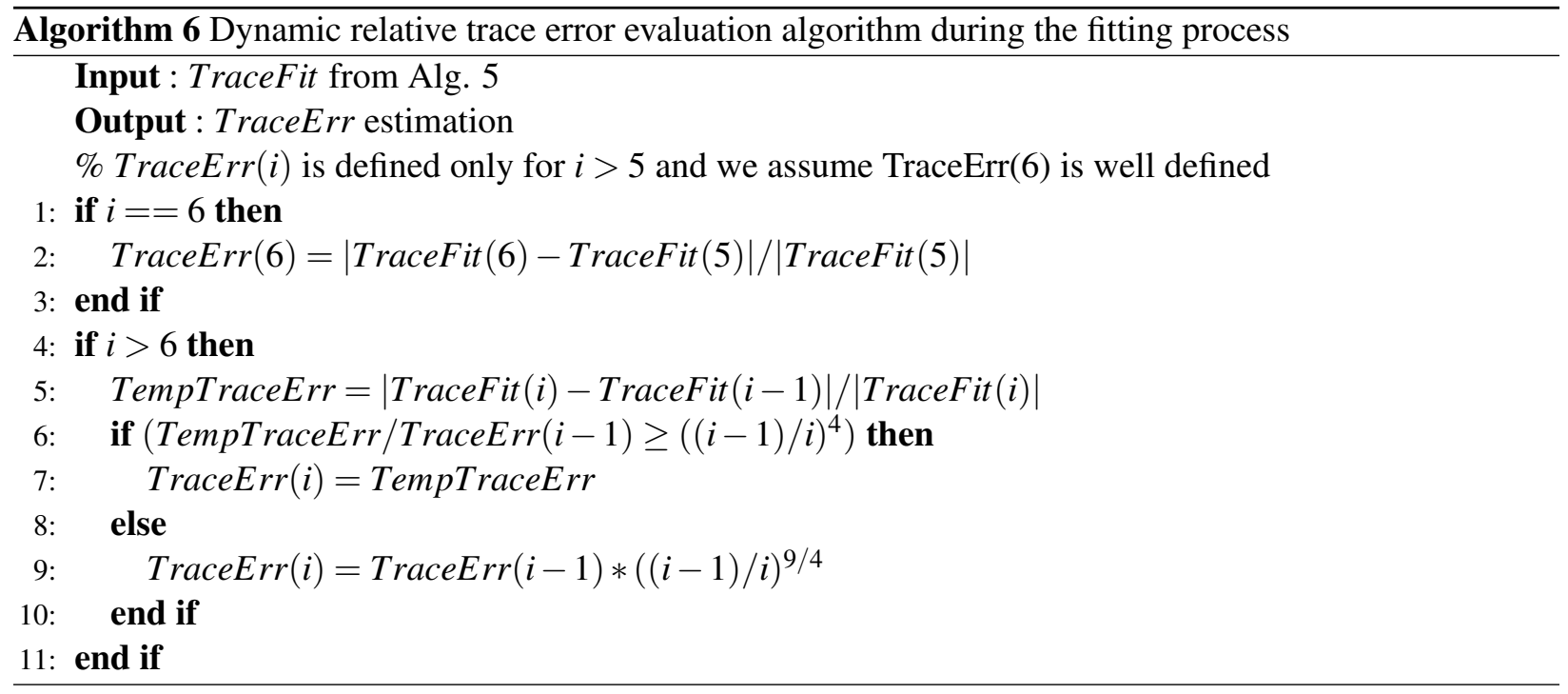

One caveat is that (21) may not be tight since the same bound holds for each subinterval $\left[\hat{M}\left(s_{j}\right), \hat{M}\left(s_{j+1}\right)\right]$. This means that a high derivative in one subinterval might dominate the bound in (21) but should not affect the error in other intervals. Therefore, convergence might not be fully dictated by (22). In practice, we found that the improvement ratio is between $O\left(k_{i} / k_{i+1}\right)$ and $O\left(\left(k_{i} / k_{i+1}\right)^{4}\right)$. Therefore, if the current relative trace error estimate is determined not to be trusted, we may instead use $\left|E_{i+1}(\hat{M})\right| \approx\left(k_{i} / k_{i+1}\right)^{k}\left|E_{1}(\hat{M})\right|, k \in[1,4]$. The choice of $k$ depends on the quality of $M$. In our experiments, we use the geometric mean of the four rates, yielding $k=9 / 4$. Recall that the corresponding ratio in $\mathrm{MC}$ is $O\left(\sqrt{k_{i} / k_{i+1}}\right)$, which is much slower than the proposed trace estimation method.

Algorithm 6 combines the two heuristics in (20) and (22) to dynamically monitor the relative trace error. It is called after step 12 of Algorithm 5. Figure 7 shows two examples of how our dynamic method provides reasonable estimates of the relative trace error.

\section{Numerical Experiments}

We run experiments on matrices that are sufficiently large to avoid misleading results due to sampling from small spaces but can still be inverted to obtain the exact trace error. We select matrices RDB5000 and cfd1 from the University of Florida sparse matrix collection [37] and generate three test matrices from applications that appear in [7]. The Heatflow160 matrix is from the discretization of the linear heat flow 


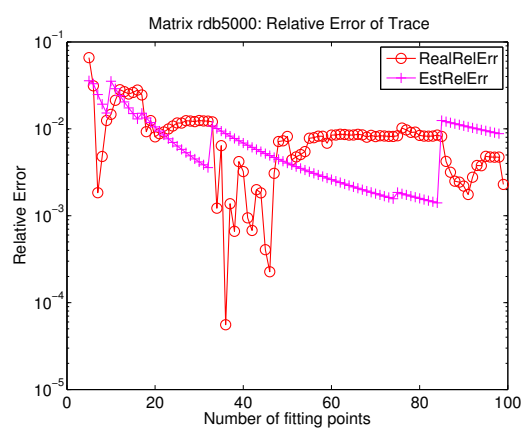

(a) Monitoring relative trace error with ILU

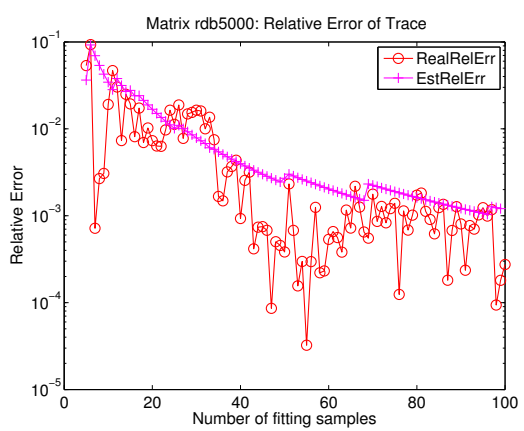

(b) Monitoring relative trace error with SVD

Figure 7: Two examples of monitoring relative trace error of the matrix RDB5000 with ILU and SVD respectively.

problem using the simplest implicit finite difference method. The matrix Poisson 150 is from 5-point central difference discretization of the 2D Poisson's equation on a square mesh. The VFH6 matrix is from the transverse vibration of a Vicsek fractal that is constructed self-similarly. We also use matrix matb5 which is a discretization of the Wilson Dirac operator on a $8^{4}$ uniform lattice with 12 degrees of freedom at each node, using a mass near to critical.

Table 2 lists these matrices along with some of their basic properties. All experiments are conducted using MATLAB 2013a. The number of fitting points increases as $s=5: 100$. The approximation $M$ is computed by ILU with parameters type $=$ ilutp and droptol $=1 \mathrm{E}-2$, or as a low rank approximation of $2 s$ smallest singular vectors with accuracy $1 \mathrm{E}-6$ (twice the number of fitting points at each step), or by the bounds on the diagonal.

Table 2: Basic information of the test matrices

\begin{tabular}{|c|c|c|c|c|}
\hline Matrix & Order & $\mathrm{nnz}(\mathrm{A})$ & $\kappa(A)$ & Application \\
\hline RDB5000 & 5000 & 29600 & $1.7 \mathrm{E} 3$ & computational fluid \\
\hline cfd1 & 70656 & 1825580 & $1.8 \mathrm{E} 7$ & computational fluid \\
\hline Heatflow160 & 25600 & 127360 & $2.6 \mathrm{E} 0$ & linear heat flow \\
\hline Poisson150 & 22500 & 111900 & $1.3 \mathrm{E} 4$ & computational fluid \\
\hline VFH6 & 15625 & 46873 & $7.2 \mathrm{E} 1$ & vicsek fractal \\
\hline matb5 & 49152 & 2359296 & $8.2 \mathrm{E} 4$ & lattice QCD \\
\hline
\end{tabular}

\subsection{Effectiveness of the fitting models}

In Figure 8, we divide the diagonal elements of the matrix RDB5000 into three contiguous sets and zoom in the details. We see that despite a good $M$, the linear LS model cannot scale the entire $M$ onto $D$. The more flexible piecewise approach of PCHIP results in a much better fit.

In Figure 9, we look at three matrices with $M$ generated using the SVD. The PCHIP model typically has smaller relative trace error than the LS model. We also see that as more fitting points are sampled, the relative trace error of both models decreases significantly at early stages and slowly after a certain point. This relates to the quality of $M$, not of the model. Typically $M$ will approximate the global pattern of $D$ and the two can be matched well with only a few fitting points. But if the local patterns of $M$ and $D$ differ, a large number of fitting points will be required. This can be seen in Figure 10. Using the permutation of $\hat{M}$ for each case, we plot $\hat{M}, D$, and $p(\hat{M})$ using 100 fitting points. For the Heatflow160 matrix, $p(M)$ approximates $D$ well everywhere except for the small leftmost part of the plot, which allows the relative error to reach below 


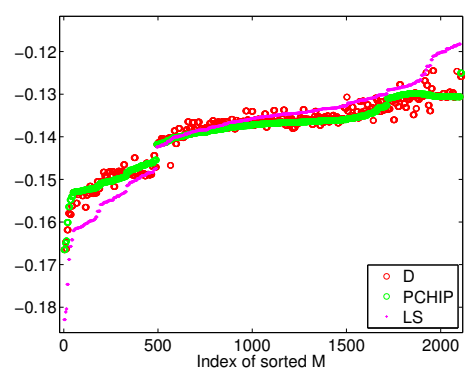

(a) Diagonal elements in $(1,2000)$

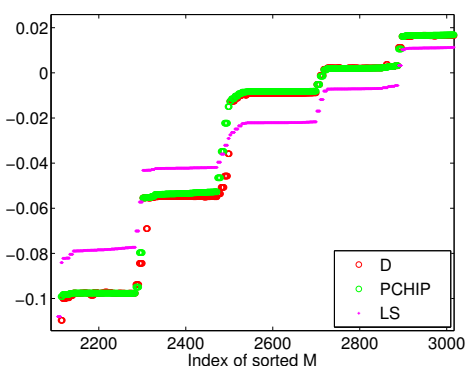

(b) Diagonal elements in $(2000,3000)$

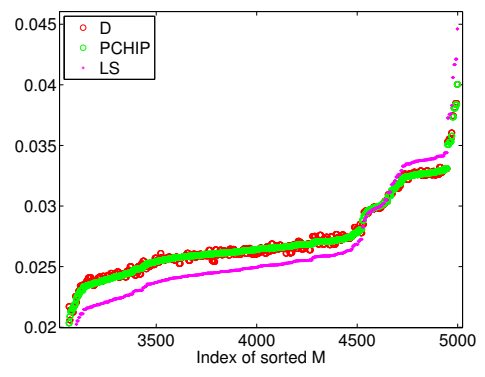

(c) Diagonal elements in $(3000,5000)$

Figure 8: Comparing the linear LS model with the PCHIP model on RDB5000 matrix with $M$ from SVD.

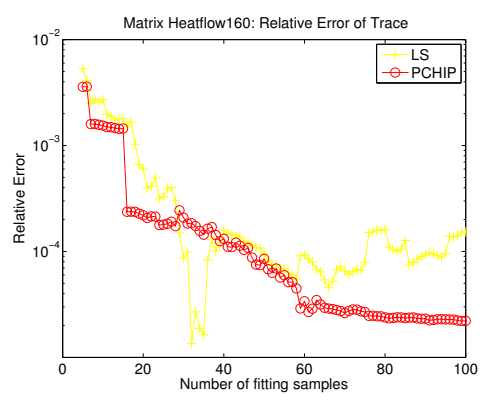

(a) Matrix Heatflow160

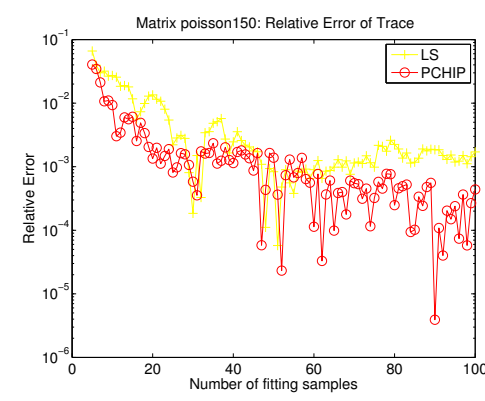

(b) Matrix Poisson150

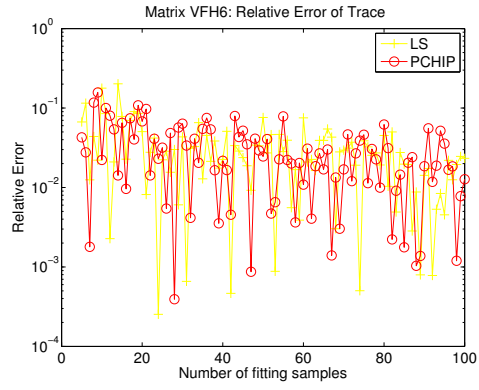

(c) Matrix VFH6

Figure 9: Comparing relative trace error between the LS model and the PCHIP model in three typical cases with SVD.

$10^{-4}$ before convergence slows down (Figure 9). The behavior is similar for the Poisson150. The issue is more pronounced on matrix VFH6, where $M$ and $p(M)$ capture the average location of $D$ but completely miss the local pattern, which is reflected by a very slowly improving error in Figure 9.

We mention that the irregularity of the relative trace errors in Figure 9 relates to the variability of successive updates of $M$ and of the sampling indices, especially when $M$ is of lower quality.

Figure 11 demonstrates that the PCHIP model has smaller actual variance for MC on $E_{f i t}$ than the LS model. Therefore, we only consider the PCHIP model in the rest of experiments.

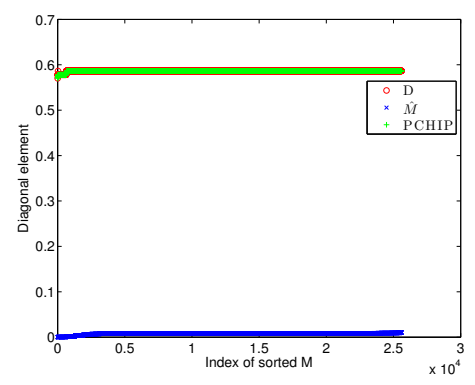

(a) Matrix Heatflow160

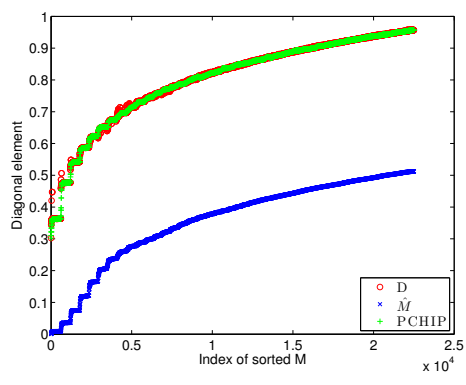

(b) Matrix Poisson 150

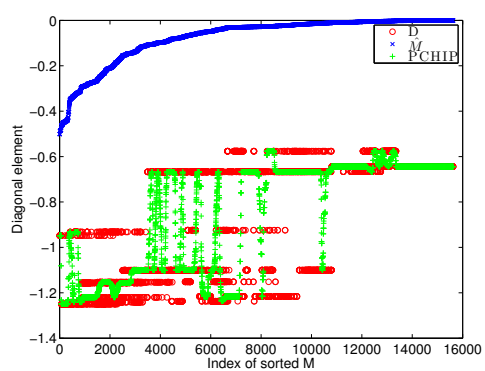

(c) Matrix VFH6

Figure 10: Fitting results of three typical cases with the PCHIP model and 100 fitting points using SVD. 


\subsection{Comparison between the fitting model and different MC methods}

We address the question of whether the number of matrix inversions we spend on computing the fitting could have been used more efficiently in an MC method, specifically the Hutchinson method on $A^{-1}$ and the Hutchinson method on $E$. In Table 3 we compare the relative trace error of the PCHIP model with 20 fitting points against the relative errors of the two MC methods as computed explicitly from their respective standard deviations in (2) and (7), with $s=20$, divided by the actual trace of $D$.

When $M$ approximates $D$ sufficiently well, the trace from the fitted diagonal is better, and for the ILU approximations far better, than if we just use the Hutchinson method on $A^{-1}$ (the first column of results). Although MC on $E$ exploits the ILU or SVD approximation of the entire matrix (not just the diagonal that our method uses), we see that it does not always improve on $\mathrm{MC}$ on $A^{-1}$, and in some cases (cfd1 with ILU) it is far worse. In contrast, our diagonal fitting typically improves on $\mathrm{MC}$ on $E$. The last column shows that even with an inexpensive diagonal approximation we obtain a similar or better error than $\mathrm{MC}$ on $A^{-1}$. The only exception is the matrix VFH6 where, as we saw earlier, $M$ cannot capture the pattern of $D$. Even then, its error is close to the errors from the MC methods and, as we show next, the best method can be identified dynamically with only a small number of samples.

Table 3: Relative trace error from our PCHIP model and from the MC method on $A^{-1}$ and on $E$ (computed explicitly as the standard deviation with $s=20$ from (2) and (7) divided by the actual trace).

\begin{tabular}{|c|c|cc|cc|c|}
\hline \multicolumn{2}{|l|}{} & \multicolumn{2}{|c|}{ ILU } & \multicolumn{2}{c|}{ SVD } & Bounds \\
\hline Matrix & $T_{Z_{2}}\left(A^{-1}\right)$ & PCHIP & $T_{Z_{2}}(E)$ & PCHIP & $T_{Z_{2}}(E)$ & PCHIP \\
\hline RDB5000 & $5.2 \mathrm{E}-2$ & $8.1 \mathrm{E}-3$ & $4.8 \mathrm{E}-2$ & $4.1 \mathrm{E}-3$ & $1.2 \mathrm{E}-2$ & $5.3 \mathrm{E}-2$ \\
\hline cfd1 & $1.3 \mathrm{E}-1$ & $2.8 \mathrm{E}-2$ & $8.2 \mathrm{E}+2$ & $8.8 \mathrm{E}-3$ & $1.8 \mathrm{E}-2$ & $2.6 \mathrm{E}-2$ \\
\hline Heatflow160 & $4.9 \mathrm{E}-4$ & $1.6 \mathrm{E}-7$ & $4.0 \mathrm{E}-5$ & $2.0 \mathrm{E}-4$ & $4.9 \mathrm{E}-4$ & $3.5 \mathrm{E}-4$ \\
\hline Poisson150 & $2.6 \mathrm{E}-2$ & $2.3 \mathrm{E}-3$ & $2.5 \mathrm{E}-2$ & $1.4 \mathrm{E}-3$ & $4.3 \mathrm{E}-3$ & $8.3 \mathrm{E}-3$ \\
\hline VFH6 & $3.2 \mathrm{E}-3$ & $6.8 \mathrm{E}-5$ & $3.1 \mathrm{E}-5$ & $1.0 \mathrm{E}-2$ & $3.2 \mathrm{E}-3$ & $6.0 \mathrm{E}-2$ \\
\hline
\end{tabular}

If the user requires better trace accuracy than our fitting technique provides, we explore the performance of the diagonal fitting as a variance reduction for MC with unit vectors. We compute the actual values of $\operatorname{Var}\left(T_{e_{i}}\left(E_{f i t}\right)\right), \operatorname{Var}\left(T_{e_{i}}\left(A^{-1}\right)\right), \operatorname{Var}\left(T_{e_{i}}(E)\right), \operatorname{Var}\left(T_{Z_{2}}\left(A^{-1}\right)\right)$ and $\operatorname{Var}\left(T_{Z_{2}}(E)\right)$ for every step $s=5: 100$ and show results for three matrices in Figure 11. Note that the low rank approximation uses $2 s$ singular vectors. As before, for Heatflow160, MC on $E_{\text {fit }}$ performs much better than other MC methods, achieving about two orders reduction in variance. For Poisson 150, MC on $E_{f i t}$ is slightly better compared to the Hutchinson method on $E$. In contrast, for VFH6, MC with unit vectors do not perform well regardless of the diagonal.

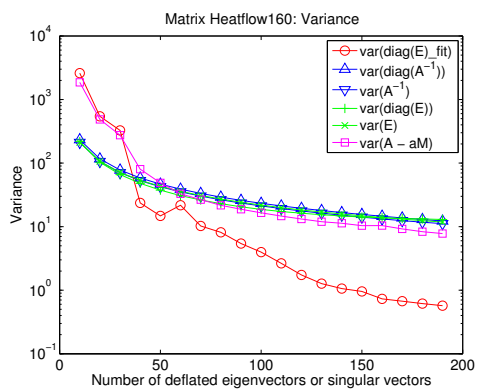

(a) Matrix Heatflow160

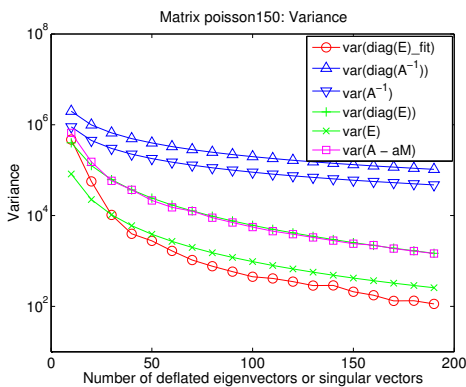

(b) Matrix Poisson150

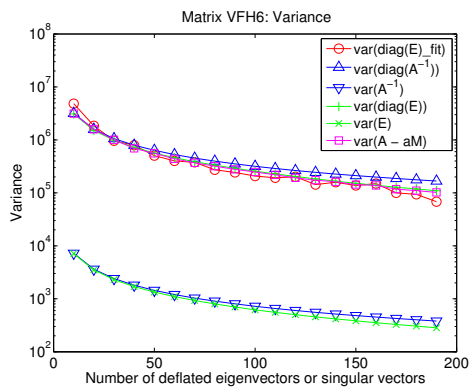

(c) Matrix VFH6

Figure 11: Comparing actual variances of different MC methods in three typical cases with SVD. 


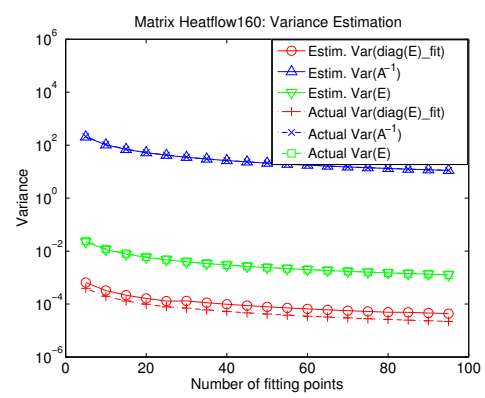

(a) Matrix Heatflow160

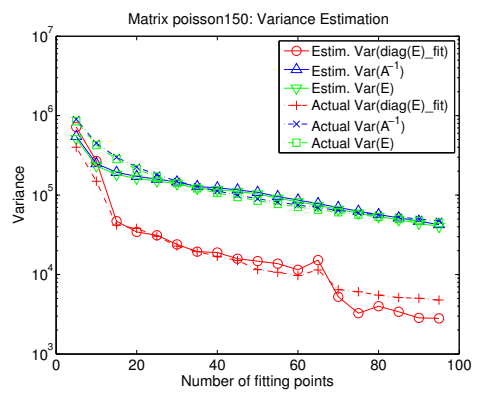

(b) Matrix Poisson 150

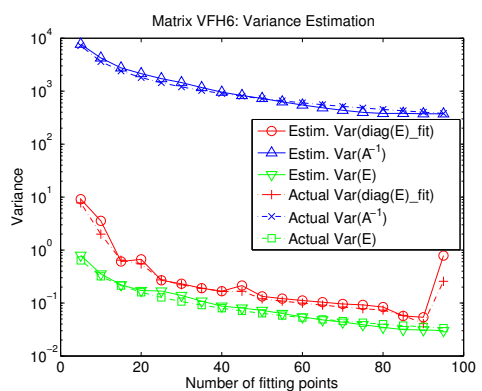

(c) Matrix VFH6

Figure 12: Comparing estimated variances and actual variances of three MC methods with ILU.

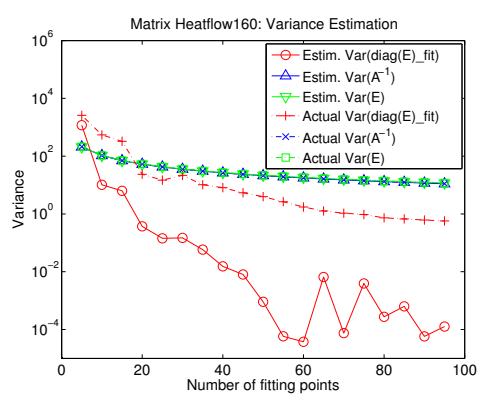

(a) Matrix Heatflow160

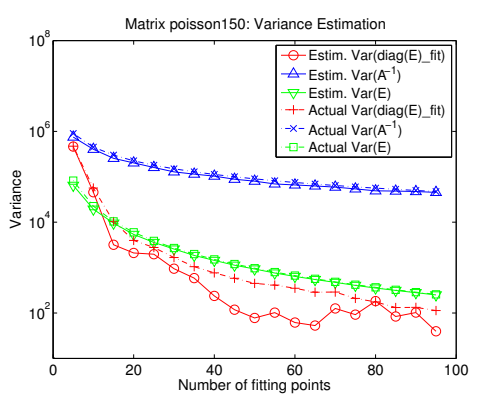

(b) Matrix Poisson150

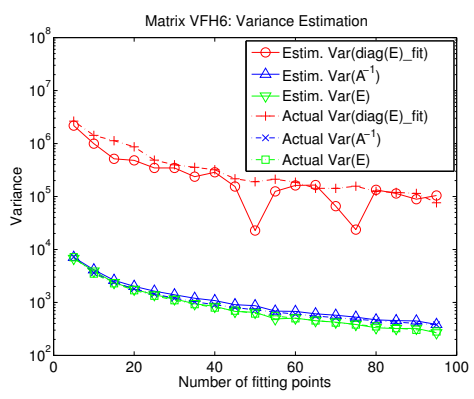

(c) Matrix VFH6

Figure 13: Comparing estimated variances and actual variances of three MC methods with SVD.

\subsection{Dynamic evaluation of variance and relative trace error}

The above results emphasize the importance of being able to assess quickly and accurately the relative differences between the variances of different methods as well as the trace error, so that we can decide whether to continue with fitting or which MC method to switch to. First we show the effectiveness of the dynamic variance evaluation algorithm for our fitting MC method on $(D-p(M))$ with unit vectors, and on $A^{-1}$ and $E$ with Rademacher vectors. Then, we evaluate our algorithm for estimating the relative trace error during the fitting process.

Figure 12 compares the estimated variances with the actual variances of the three MC methods when increasing the number of fitting points from 5 to 100 . The approximation $M$ is computed by using ILU. We can see that the estimated values of $\operatorname{Var}\left(T_{Z_{2}}\left(A^{-1}\right)\right)$ and $\operatorname{Var}\left(T_{Z_{2}}(E)\right)$ converge to the actual variances after only a few sample points. The estimated value of $\operatorname{Var}\left(T_{e_{i}}\left(E_{f i t}\right)\right)$ gets close to and captures the trend of the the actual variance as the fitting samples increase. Nevertheless, the relative differences between the variances of the various MC methods are apparent almost immediately.

Figure 13 shows the same experiments when the approximation $M$ is computed by SVD. Since $M$ is updated each step, $\operatorname{Var}\left(T_{Z_{2}}(E)\right)$ and $\operatorname{Var}\left(T_{e_{i}}\left(E_{f i t}\right)\right)$ change accordingly. As with ILU, $\operatorname{Var}\left(T_{Z_{2}}\left(A^{-1}\right)\right)$ and $\operatorname{Var}\left(T_{Z_{2}}(E)\right.$ can be estimated very well in a few steps. $\operatorname{Var}\left(T_{e_{i}}\left(E_{f i t}\right)\right)$ could be underestimated but the relative variance difference between these MC methods becomes clear when the fitting points increase beyond 20. Thus we are able to determine whether the fitting process is beneficial as a variance reduction preprocessing and which is the best MC method to proceed with for the trace estimation.

Figure 14 compares the estimated relative trace error with the actual one in the cases of Figure 13. We observe that the estimation is accurate as the fitting samples increase, even for cases such as VFH6 where the fitting process is not as successful. Moreover, because our algorithm is based on upper bounds on the error of a piecewise cubic polynomial, the actual relative trace error could be lower than predicted. 


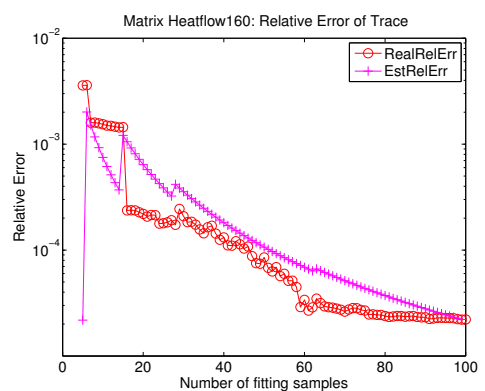

(a) Matrix Heatflow160

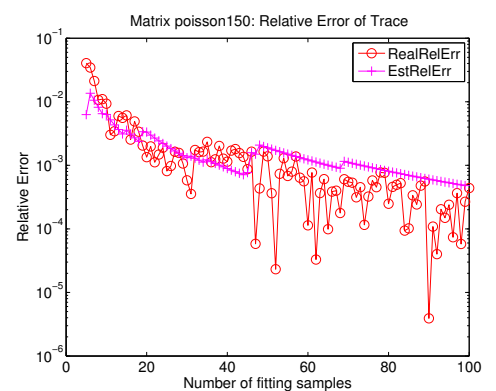

(b) Matrix Poisson 150

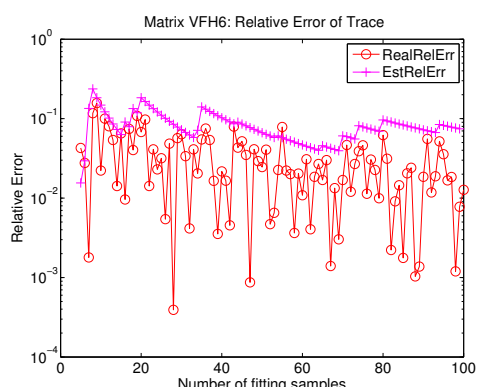

(c) Matrix VFH6

Figure 14: Comparing estimated relative trace error with actual relative trace error with SVD.

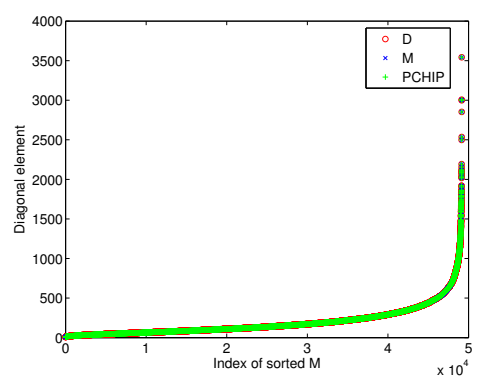

(a) PCHIP results

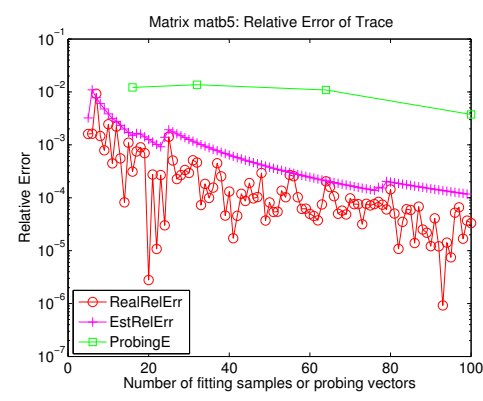

(b) Relative trace error

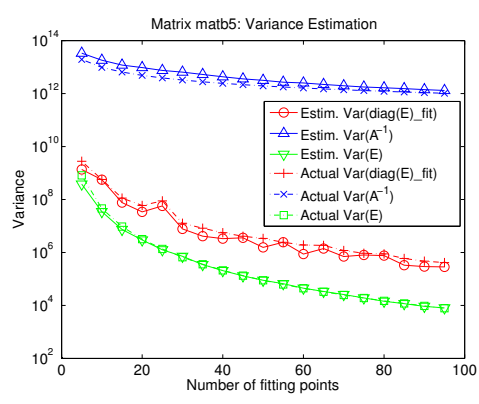

(c) Variances

Figure 15: Fitting results, dynamic evaluation of relative trace error and variances with SVD on a large QCD matrix. In Figure 15(b), the green square denotes the relative trace error by applying hierarchical probing technique on deflated matrix $E$ in [4].

\subsection{A large $Q C D$ problem}

The trace estimator presented in this paper has the potential of improving a number of LQCD calculations, where the trace of the Dirac matrix is related to an important property of QCD called spontaneous chiral symmetry breaking [23]. The authors in [4] presented the method of hierarchical probing that achieves almost optimal variance reduction incrementally and inexpensively on regular lattices.

As shown in Figure 15(a), a low rank approximation with 200 singular vectors yields a good approximation $M$ and an excellent fit $p(M)$. In Figure 15(b), we see that the actual relative trace error decreases very fast to the order of $1 e-4$ with increasing number of fitting points and singular vectors, and can be monitored well by our dynamic trace estimation algorithm. These singular vectors can be approximated while solving the linear systems with eigCG. Interestingly, we can improve the relative trace error of hierarchical probing by two orders of magnitude. In addition, the variances of different MC methods can be estimated dynamically to allow us to continue with the best estimator if needed (Figure 15(c)).

\section{Conclusion and future work}

A novel method has been presented to estimate the trace of the matrix inverse by exploiting the pattern correlation between the diagonal of the inverse of the matrix and some approximation. The key idea is to construct a good approximation $M \approx D$ through eigenvectors or some preconditioner, sample important patterns of $D$ by using the distribution of the elements of $M$, and use fitting techniques to obtain a better approximation $p(M) \approx D$ from where we obtain a trace estimate. The proposed method can provide a fast trace estimate with 2-3 digits relative accuracy given only a few samples while may or may not improve the 
variance of MC. When the variance is reduced sufficiently, our method can be also used as a diagonal estimator. We also propose an effective dynamic variance evaluation algorithm to determine the MC method with the smallest variance and a dynamic relative trace error estimation algorithm without any additional costs. We demonstrated the effectiveness of these methods through a set of experiments in some real applications.

\section{Acknowledgement}

The authors thank Professor Yousef Saad for his helpful comments and discussions to improve the manuscript. The authors also thank reviewers' valuable comments to improve the quality and readability of the manuscript. This work is supported by NSF under grants No. CCF 1218349 and ACI SI2-SSE 1440700, and by DOE under a grant No. DE-FC02-12ER41890.

\section{References}

[1] M. F. Hutchinson, A stochastic estimator of the trace of the influence matrix for laplacian smoothing splines, Communications in Statistics-Simulation and Computation 19 (2) (1990) 433-450.

[2] C. Bekas, A. Curioni, I. Fedulova, Low cost high performance uncertainty quantification, in: Proceedings of the 2nd Workshop on High Performance Computational Finance, ACM, 2009, p. 8.

[3] V. Kalantzis, C. Bekas, A. Curioni, E. Gallopoulos, Accelerating data uncertainty quantification by solving linear systems with multiple right-hand sides, Numerical Algorithms 62 (4) (2013) 637-653.

[4] A. Stathopoulos, J. Laeuchli, K. Orginos, Hierarchical probing for estimating the trace of the matrix inverse on toroidal lattices, SIAM Journal on Scientific Computing 35 (5) (2013) S299-S322.

[5] H. Avron, Counting triangles in large graphs using randomized matrix trace estimation, in: Workshop on Large-scale Data Mining: Theory and Applications, 2010.

[6] H. Avron, S. Toledo, Randomized algorithms for estimating the trace of an implicit symmetric positive semi-definite matrix, Journal of the ACM 58 (2) (2011) 8.

[7] Z. Bai, G. Fahey, G. Golub, Some large-scale matrix computation problems, Journal of Computational and Applied Mathematics 74 (1) (1996) 71-89.

[8] I. S. Duff, A. M. Erisman, J. K. Reid, Direct methods for sparse matrices, Clarendon Press Oxford, 1986.

[9] J. Xia, Y. Xi, S. Cauley, V. Balakrishnan, Fast sparse selected inversion, SIAM Journal on Matrix Analysis and Applications 36 (3) (2015) 1283-1314.

[10] J. M. Tang, Y. Saad, Domain-decomposition-type methods for computing the diagonal of a matrix inverse, SIAM Journal on Scientific Computing 33 (5) (2011) 2823-2847.

[11] H. Guo, Computing traces of functions of matrices, A Journal of Chinese Universities (English series) 2 (2000) 204-215.

[12] G. Meurant, Estimates of the trace of the inverse of a symmetric matrix using the modified chebyshev algorithm, Numerical Algorithms 51 (3) (2009) 309-318.

[13] C. Brezinski, P. Fika, M. Mitrouli, Moments of a linear operator, with applications to the trace of the inverse of matrices and the solution of equations, Numerical Linear Algebra with Applications 19 (6) (2012) 937-953. 
[14] M. N. Wong, F. J. Hickernell, K. I. Liu, Computing the trace of a function of a sparse matrix via hadamard-like sampling, Tech. rep., Department of Mathematics, Hong Kong Baptist University (2004).

[15] C. Bekas, E. Kokiopoulou, Y. Saad, An estimator for the diagonal of a matrix, Applied Numerical Mathematics 57 (11) (2007) 1214-1229.

[16] J. Chen, How accurately should i solve linear systems when applying the hutchinson trace estimator?, Tech. rep., IBM Thomas J. Watson Research Center (2015).

[17] F. Roosta-Khorasani, U. Ascher, Improved bounds on sample size for implicit matrix trace estimators, Foundations of Computational Mathematics (2014) 1-26.

[18] J. M. Tang, Y. Saad, A probing method for computing the diagonal of a matrix inverse, Numerical Linear Algebra with Applications 19 (3) (2012) 485-501.

[19] A. Erisman, W. Tinney, On computing certain elements of the inverse of a sparse matrix, Communications of the ACM 18 (3) (1975) 177-179.

[20] L. Lin, C. Yang, J. C. Meza, J. Lu, L. Ying, W. E, Selinv - an algorithm for selected inversion of a sparse symmetric matrix, ACM Trans. Math. Softw. 37 (4) (2011) 40.

[21] L. Wu, A. Stathopoulos, A preconditioned hybrid svd method for accurately computing singular triplets of large matrices, SIAM Journal on Scientific Computing 37 (5) (2015) S365-S388.

[22] L. Wu, E. Romero, A. Stathopoulos, Primme_svds: A high-performance preconditioned svd solver for accurate large-scale computations, arXiv preprint arXiv:1607.01404.

[23] A. Stathopoulos, K. Orginos, Computing and deflating eigenvalues while solving multiple right-hand side linear systems with an application to quantum chromodynamics, SIAM Journal on Scientific Computing 32 (1) (2010) 439-462.

[24] A. M. Abdel-Rehim, A. Stathopoulos, K. Orginos, Extending the eigcg algorithm to nonsymmetric lanczos for linear systems with multiple right-hand sides, Numerical Linear Algebra with Applications 21 (4) (2014) 473-493.

[25] P. D. Robinson, A. J. Wathen, Variational bounds on the entries of the inverse of a matrix, IMA journal of numerical analysis 12 (4) (1992) 463-486.

[26] D. J. MacKay, Introduction to monte carlo methods, in: Learning in graphical models, Springer, 1998, pp. 175-204.

[27] J. M. Steele, Certifying smoothness of discrete functions and measuring legitimacy of images, Journal of Complexity (5) (1989) 261-270.

[28] J. Kiefer, Optimum sequential search and approximation methods under minimum regularity assumptions, J. Soc. Indust. Appl. Math. 5 (1957) 105-136.

[29] E. Novak, Quadrature formulas for monotone functions, Proceedings of the American Mathematical Society 115 (1) (1992) 59-68.

[30] A. G. Sukharev, The concept of sequential optimality for problems in numerical analysis, Journal of Complexity 3 (3) (1987) 347-357. doi:http://dx.doi.org/10.1016/0885-064X(87)90020-3. 
[31] F. N. Fritsch, R. E. Carlson, Monotone piecewise cubic interpolation, SIAM Journal on Numerical Analysis 17 (2) (1980) 238-246.

[32] F. E. Harrell, Regression modeling strategies, Springer Science \& Business Media, 2001.

[33] S. Arlot, A. Celisse, et al., A survey of cross-validation procedures for model selection, Statistics Surveys 4 (2010) 40-79.

[34] T. Gudmundsson, C. S. Kenney, A. J. Laub, Small-sample statistical estimates for matrix norms, SIAM Journal on Matrix Analysis and Applications 16 (3) (1995) 776-792.

[35] C. Kenney, A. J. Laub, M. Reese, Statistical condition estimation for linear systems, SIAM Journal on Scientific Computing 19 (2) (1998) 566-583.

[36] T. R. Lucas, Error bounds for interpolating cubic splines under various end conditions, SIAM Journal on Numerical Analysis 11 (3) (1974) 569-584.

[37] T. A. Davis, Y. Hu, The university of florida sparse matrix collection, ACM Transactions on Mathematical Software 38 (1) (2011) 1. 